\title{
A General Iterative Method for Variational Inequality Problems, Mixed Equilibrium Problems, and Fixed Point Problems of Strictly Pseudocontractive Mappings in Hilbert Spaces
}

\author{
Rattanaporn Wangkeeree and Rabian Wangkeeree \\ Department of Mathematics, Faculty of Science, Naresuan University, Phitsanulok 65000, Thailand
}

Correspondence should be addressed to Rabian Wangkeeree, rabianw@nu.ac.th

Received 23 April 2009; Accepted 22 June 2009

Recommended by Anthony To Ming Lau

\begin{abstract}
We introduce an iterative scheme for finding a common element of the set of fixed points of a $k$ strictly pseudocontractive mapping, the set of solutions of the variational inequality for an inversestrongly monotone mapping, and the set of solutions of the mixed equilibrium problem in a real Hilbert space. Under suitable conditions, some strong convergence theorems for approximating a common element of the above three sets are obtained. As applications, at the end of the paper we first apply our results to study the optimization problem and we next utilize our results to study the problem of finding a common element of the set of fixed points of two families of finitely $k$ strictly pseudocontractive mapping, the set of solutions of the variational inequality, and the set of solutions of the mixed equilibrium problem. The results presented in the paper improve some recent results of Kim and Xu (2005), Yao et al. (2008), Marino et al. (2009), Liu (2009), Plubtieng and Punpaeng (2007), and many others.
\end{abstract}

Copyright (c) 2009 R. Wangkeeree and R. Wangkeeree. This is an open access article distributed under the Creative Commons Attribution License, which permits unrestricted use, distribution, and reproduction in any medium, provided the original work is properly cited.

\section{Introduction}

Throughout this paper, we always assume that $H$ is a real Hilbert space with inner product $\langle\cdot, \cdot\rangle$ and norm $\|\cdot\|$, respectively, $C$ is a nonempty closed convex subset of $H$. Let $\varphi: C \rightarrow \mathbb{R}$ be a real-valued function and let $\Theta: C \times C \rightarrow \mathbb{R}$ be an equilibrium bifunction, that is, $\Theta(u, u)=0$ for each $u \in C$. Ceng and Yao [1] considered the following mixed equilibrium problem:

$$
\text { Find } x^{*} \in C \text { such that } \Theta\left(x^{*}, y\right)+\varphi(y) \geq \varphi\left(x^{*}\right), \quad \forall y \in C \text {. }
$$

The set of solutions of (1.1) is denoted by $\operatorname{MEP}(\Theta, \varphi)$. It is easy to see that $x^{*}$ is a solution of problem (1.1) implies that $x^{*} \in \operatorname{dom} \varphi=\{x: \varphi(x)<+\infty\}$. 
In particular, if $\varphi \equiv 0$, the mixed equilibrium problem (1.1) becomes the following equilibrium problem:

$$
\text { Find } x^{*} \in C \text { such that } \Theta\left(x^{*}, y\right) \geq 0, \quad \forall y \in C \text {. }
$$

The set of solutions of (1.2) is denoted by $\operatorname{EP}(\Theta)$.

If $\varphi \equiv 0$ and $\Theta(x, y)=\langle B x, y-x\rangle \geq 0$ for all $x, y \in C$, where $B$ is a mapping form $C$ into $H$, then the mixed equilibrium problem (1.1) becomes the following variational inequality:

$$
\text { Find } x^{*} \in C \text { such that }\left\langle B x^{*}, y-x^{*}\right\rangle \geq 0, \quad \forall y \in C \text {. }
$$

The set of solutions of (1.3) is denoted by $\operatorname{VI}(B, C)$. The variational inequality has been extensively studied in literature. See, for example, [2-13] and the references therein.

The problem (1.1) is very general in the sense that it includes, as special cases, optimization problems, variational inequalities, minimax problems, Nash equilibrium problem in noncooperative games and others; see for instance, [1, 2, 14, 15].

First we recall some relevant important results as follows.

In 1997, Combettes and Hirstoaga [14] introduced an iterative method of finding the best approximation to the initial data when $\mathrm{EP}(\Theta)$ is nonempty and proved a strong convergence theorem. Subsequently, S. Takahashi and W. Takahashi [16] introduced an iterative scheme by the viscosity approximation method for finding a common element of the set of solutions of $\mathrm{EP}(\Theta)$ and the set of fixed point points of a nonexpansive mapping. Using the idea of S. Takahashi and W. Takahashi [16], Plubtieng and Punpaeng [17] introduced an the general iterative method for finding a common element of the set of solutions of $\operatorname{EP}(\Theta)$ and the set of fixed points of a nonexpansive mapping which is the optimality condition for the minimization problem in a Hilbert space. Furthermore, Yao et al. [11] introduced some new iterative schemes for finding a common element of the set of solutions of $\mathrm{EP}(\Theta)$ and the set of common fixed points of finitely (infinitely) nonexpansive mappings. Very recently, Ceng and Yao [1] considered a new iterative scheme for finding a common element of the set of solutions of $\operatorname{MEP}(\Theta)$ and the set of common fixed points of finitely many nonexpansive mappings in a Hilbert space and obtained a strong convergence theorem which used the following condition:

(E) $K: C \rightarrow \mathbb{R}$ is $\eta$-strongly convex and its derivative $K^{\prime}$ is sequentially continuous from the weak topology to the strong topology.

Their results extend and improve the corresponding results in $[6,11,14]$. We note that the condition (E) for the function $K: C \rightarrow \mathbb{R}$ is a very strong condition. We also note that the condition (E) does not cover the case $K(x)=\|x\|^{2} / 2$ and $\eta(x, y)=x-y$. Motivated by Ceng and Yao [1], Peng and Yao [18] introduced a new iterative scheme based on only the extragradient method for finding a common element of the set of solutions of a mixed equilibrium problem, the set of fixed points of a family of finitely nonexpansive mappings and the set of the variational inequality for a monotone Lipschitz continuous mapping. They obtained a strong convergence theorem without the condition $(E)$ for the sequences generated by these processes. 
We recall that a mapping $B: C \rightarrow H$ is said to be:

(i) monotone if $\langle B x-B y, x-y\rangle \geq 0$, for all $x, y \in C$,

(ii) $L$-Lipschitz if there exists a constant $L>0$ such that $\|B x-B y\| \leq L \| x-$ $y \|$, for all $x, y \in C$,

(iii) $\alpha$-inverse-strongly monotone $[19,20]$ if there exists a positive real number $\alpha$ such that

$$
\langle B x-B y, x-y\rangle \geq \alpha\|B x-B y\|^{2}, \quad \forall x, y \in C .
$$

It is obvious that any $\alpha$-inverse-strongly monotone mapping $B$ is monotone and Lipschitz continuous. Recall that a mapping $T: C \rightarrow C$ is called a $k$-strictly pseudocontractive mapping if there exists a constant $0 \leq k<1$ such that

$$
\|T x-T y\|^{2} \leq\|x-y\|^{2}+k\|(I-T) x-(I-T) y\|^{2}, \quad \forall x, y \in C
$$

Note that the class of $k$-strictly pseudocontractive mappings strictly includes the class of nonexpansive mappings which are mappings $T$ on $C$ such that

$$
\|T x-T y\| \leq\|x-y\|, \quad \forall x, y \in C .
$$

That is, $T$ is nonexpansive if and only if $T$ is 0 -strictly pseudocontractive. We denote by $F(T):=\{x \in C: T x=x\}$ the set of fixed points of $T$.

Iterative methods for nonexpansive mappings have recently been applied to solve convex minimization problems; see, for example, [21-24] and the references therein. Convex minimization problems have a great impact and influence in the development of almost all branches of pure and applied sciences. A typical problem is to minimize a quadratic function over the set of the fixed points of nonexpansive mapping on a real Hilbert space:

$$
\theta(x)=\min _{x \in C} \frac{1}{2}\langle A x, x\rangle-\langle x, b\rangle,
$$

where $A$ is a linear bounded operator, $C$ is the fixed point set of a nonexpansive mapping $T$, and $b$ is a given point in $H$. Recall that a linear bounded operator $A$ is strongly positive if there is a constant $\bar{\gamma}>0$ with property

$$
\langle A x, x\rangle \geq \bar{\gamma}\|x\|^{2} \quad \forall x \in H .
$$

Recently, Marino and Xu [25] introduced the following general iterative scheme based on the viscosity approximation method introduced by Moudafi [26]:

$$
x_{n+1}=\left(I-\alpha_{n} A\right) T x_{n}+\alpha_{n} \gamma f\left(x_{n}\right), \quad n \geq 1,
$$


where $A$ is a strongly positive bounded linear operator on $H$. They proved that if the sequence $\left\{\alpha_{n}\right\}$ of parameters satisfies appropriate conditions, then the sequence $\left\{x_{n}\right\}$ generated by (1.9) converges strongly to the unique solution of the variational inequality

$$
\left\langle(A-\gamma f) x^{*}, x-x^{*}\right\rangle \geq 0, \quad x \in C,
$$

which is the optimality condition for the minimization problem

$$
\min _{x \in C} \frac{1}{2}\langle A x, x\rangle-h(x)
$$

where $h$ is a potential function for $\gamma f$ (i.e., $h^{\prime}(x)=\gamma f(x)$ for $x \in H$ ).

Recall that the construction of fixed points of nonexpansive mappings via Manns algorithm [27] has extensively been investigated in literature; see, for example [27-32] and references therein. If $T$ is a nonexpansive self-mapping of $C$, then Mann's algorithm generates, initializing with an arbitrary $x_{1} \in C$, a sequence according to the recursive manner

$$
x_{n+1}=\alpha_{n} x_{n}+\left(1-\alpha_{n}\right) T x_{n}, \quad \forall n \geq 1,
$$

where $\left\{\alpha_{n}\right\}$ is a real control sequence in the interval $(0,1)$.

If $T: C \rightarrow C$ is a nonexpansive mapping with a fixed point and if the control sequence $\left\{\alpha_{n}\right\}$ is chosen so that $\sum_{n=1}^{\infty} \alpha_{n}\left(1-\alpha_{n}\right)=\infty$, then the sequence $\left\{x_{n}\right\}$ generated by Manns algorithm converges weakly to a fixed point of $T$. Reich [33] showed that the conclusion also holds good in the setting of uniformly convex Banach spaces with a Fréhet differentiable norm. It is well known that Reich's result is one of the fundamental convergence results. However, this scheme has only weak convergence even in a Hilbert space [34]. Therefore, many authors try to modify normal Mann's iteration process to have strong convergence; see, for example, [35-40] and the references therein.

$\mathrm{Kim}$ and $\mathrm{Xu}[36]$ introduced the following iteration process:

$$
\begin{gathered}
y_{n}=\beta_{n} x_{n}+\left(1-\beta_{n}\right) T x_{n}, \\
x_{n+1}=\alpha_{n} u+\left(1-\alpha_{n}\right) y_{n}, \quad n \geq 1,
\end{gathered}
$$

where $T$ is a nonexpansive mapping of $C$ into itself and $u \in C$ is a given point. They proved the sequence $\left\{x_{n}\right\}$ defined by (1.13) strongly converges to a fixed point of $T$ provided the control sequences $\left\{\alpha_{n}\right\}$ and $\left\{\beta_{n}\right\}$ satisfy appropriate conditions.

In [41], Yao et al. also modified iterative algorithm (1.13) to have strong convergence by using viscosity approximation method. To be more precisely, they considered the following iteration process:

$$
\begin{gathered}
y_{n}=\beta_{n} x_{n}+\left(1-\beta_{n}\right) T x_{n}, \\
x_{n+1}=\alpha_{n} f\left(x_{n}\right)+\left(1-\alpha_{n}\right) y_{n}, \quad n \geq 1,
\end{gathered}
$$


where $T$ is a nonexpansive mapping of $C$ into itself and $f$ is an $\beta$-contraction. They proved the sequence $\left\{x_{n}\right\}$ defined by (1.14) strongly converges to a fixed point of $T$ provided the control sequences $\left\{\alpha_{n}\right\}$ and $\left\{\beta_{n}\right\}$ satisfy appropriate conditions.

Very recently, motivated by Acedo and Xu [35], Kim and Xu [36], Marino and Xu [42], and Yao et al. [41], Marino et al. [43] introduced a composite iteration scheme as follows:

$$
\begin{gathered}
y_{n}=\beta_{n} x_{n}+\left(1-\beta_{n}\right) T x_{n}, \\
x_{n+1}=\alpha_{n} \gamma f\left(x_{n}\right)+\left(I-\alpha_{n} A\right) y_{n}, \quad n \geq 1,
\end{gathered}
$$

where $T$ is a $k$-strictly pseudocontractive mapping on $H, f$ is an $\beta$-contraction, and $A$ is a linear bounded strongly positive operator. They proved that the iterative scheme $\left\{x_{n}\right\}$ defined by (1.15) converges to a fixed point of $T$, which is a unique solution of the variational inequality (1.10) and is also the optimality condition for the minimization problem provided $\left\{\alpha_{n}\right\}$ and $\left\{\beta_{n}\right\}$ are sequences in $[0,1]$ satifies the following control conditions:

(C1) $\lim _{n \rightarrow \infty} \alpha_{n}=0, \sum_{n=1}^{\infty} \alpha_{n}=\infty, \sum_{n=1}^{\infty}\left|\alpha_{n+1}-\alpha_{n}\right|<\infty$,

(C2) $0 \leq k \leq \beta_{n}<\varepsilon<1$ for all $n \geq 0$ and $\sum_{n=1}^{\infty}\left|\beta_{n+1}-\beta_{n}\right|<\infty$.

Moreover, for finding a common element of the set of fixed points of a $k$-strictly pseudocontractive nonself mapping and the set of solutions of an equilibrium problem in a real Hilbert space, Liu [44] introduced the following iterative scheme:

$$
\begin{gathered}
x_{1}=x \in C \text { chosen arbitrarily, } \\
\Theta\left(u_{n}, y\right)+\frac{1}{r_{n}}\left\langle y-u_{n}, u_{n}-x_{n}\right\rangle \geq 0, \quad \forall y \in C, \\
y_{n}=\beta_{n} u_{n}+\left(1-\beta_{n}\right) T u_{n}, \\
x_{n+1}=\alpha_{n} \gamma f\left(x_{n}\right)+\left(I-\alpha_{n} A\right) y_{n}, \quad n \geq 1,
\end{gathered}
$$

where $T$ is a $k$-strictly pseudocontractive mapping on $H, f$ is an $\alpha$-contraction and, $A$ is a linear bounded strongly positive operator. They proved that the iterative scheme $\left\{x_{n}\right\}$ defined by (1.16) converges to a common element of $F(T) \cap \mathrm{EP}(\Theta)$, which solves some variation inequality problems provided $\left\{\alpha_{n}\right\},\left\{\beta_{n}\right\}$, and $\left\{r_{n}\right\}$ are sequences in $[0,1]$ satifies the control conditions (C1) and the following conditions:

(C'2) $0 \leq k \leq \beta_{n}<\varepsilon<1$ for all $n \geq 1, \lim _{n \rightarrow \infty} \beta_{n}=\varepsilon$, and $\sum_{n=1}^{\infty}\left|\beta_{n+1}-\beta_{n}\right|<\infty$;

(C3) $\liminf _{n \rightarrow \infty} r_{n}>0, \sum_{n=1}^{\infty}\left|r_{n+1}-r_{n}\right|<0$.

All of the above bring us the following conjectures?

Question 1. (i) Could we weaken or remove the control condition $\sum_{n=1}^{\infty}\left|\alpha_{n+1}-\alpha_{n}\right|<\infty$ on parameter $\left\{\alpha_{n}\right\}$ in $(\mathrm{C} 1)$ ?

(ii) Could we weaken or remove the control condition $\sum_{n=1}^{\infty}\left|\beta_{n+1}-\beta_{n}\right|<\infty$ on parameter $\left\{\beta_{n}\right\}$ in $(\mathrm{C} 2)$ and $\left(\mathrm{C}^{\prime} 2\right)$ ?

(iii) Could we weaken or remove the control condition $\lim _{n \rightarrow \infty} \beta_{n}=\varepsilon$ on the parameter $\left\{\beta_{n}\right\}$ in $\left(C^{\prime} 2\right)$ ?

(iv) Could we weaken the control condition (C3) on parameters $\left\{r_{n}\right\}$ ? 
(v) Could we construct an iterative algorithm to approximate a common element of $F(T) \cap \operatorname{VI}(B, C) \cap \operatorname{MEP}(\Theta, \varphi)$ ?

It is our purpose in this paper that we suggest and analyze an iterative scheme for finding a common element of the set of fixed points of a $k$-strictly pseudocontractive mapping, the set of solutions of a variational inequality and the set of solutions of a mixed equilibrium problem in the framework of a real Hilbert space. Then we modify our iterative scheme to finding a common element of the set of common fixed points of two finite families of $k$-strictly pseudocontractive mappings, the set of solutions of a variational inequality and the set of solutions of a mixed equilibrium problem. Application to optimization problems which is one of the motivation in this paper is also given. The results in this paper generalize and improve some well-known results in [17, 36, 41, 43, 44].

\section{Preliminaries}

Let $H$ be a real Hilbert space with norm $\|\cdot\|$ and inner product $\langle\cdot, \cdot\rangle$ and let $C$ be a closed convex subset of $H$. We denote weak convergence and strong convergence by notations and $\rightarrow$, respectively. It is well known that for any $\lambda \in[0,1]$,

$$
\|\lambda x+(1-\lambda) y\|^{2}=\lambda\|x\|^{2}+(1-\lambda)\|y\|^{2}-\lambda(1-\lambda)\|x-y\|^{2}, \quad \forall x, y \in H
$$

For every point $x \in H$, there exists a unique nearest point in $C$, denoted by $P_{C} x$, such that

$$
\left\|x-P_{C} x\right\| \leq\|x-y\| \quad \forall y \in C
$$

$P_{C}$ is called the metric projection of $H$ onto $C$. It is well known that $P_{C}$ is a nonexpansive mapping of $H$ onto $C$ and satisfies

$$
\left\langle x-y, P_{C} x-P_{C} y\right\rangle \geq\left\|P_{C} x-P_{C} y\right\|^{2}
$$

for every $x, y \in H$. Moreover, $P_{C} x$ is characterized by the following properties: $P_{C} x \in C$ and

$$
\begin{gathered}
\left\langle x-P_{C} x, y-P_{C} x\right\rangle \leq 0, \\
\|x-y\|^{2} \geq\left\|x-P_{C} x\right\|^{2}+\left\|y-P_{C} x\right\|^{2},
\end{gathered}
$$

for all $x \in H, y \in C$. It is easy to see that the following is true:

$$
u \in \mathrm{VI}(B, C) \Longleftrightarrow u=P_{C}(u-\lambda B u), \quad \lambda>0
$$

A set-valued mapping $S: H \rightarrow 2^{H}$ is called monotone if for all $x, y \in H, f \in S x$ and $g \in S y$ imply $\langle x-y, f-g\rangle \geq 0$. A monotone mapping $S: H \rightarrow 2^{H}$ is maximal if the graph of $G(S)$ of $S$ is not properly contained in the graph of any other monotone mapping. It is known that a monotone mapping $S$ is maximal if and only if for $(x, f) \in H \times H,\langle x-y, f-g\rangle \geq 0$ for 
every $(y, g) \in G(S)$ implies $f \in S x$. Let $B$ be a monotone map of $C$ into $H$ and let $N_{C} v$ be the normal cone to $C$ at $v \in C$, that is, $N_{C} v=\{w \in H:\langle u-v, w\rangle \geq 0, \forall u \in C\}$ and define

$$
S v= \begin{cases}B v+N_{C} v, & v \in C, \\ \emptyset, & v \notin C .\end{cases}
$$

Then $S$ is the maximal monotone and $0 \in S v$ if and only if $v \in \operatorname{VI}(B, C)$; see [45].

The following lemmas will be useful for proving the convergence result of this paper.

Lemma 2.1 ([46]). Assume $\left\{a_{n}\right\}$ is a sequence of nonnegative real numbers such that

$$
a_{n+1} \leq\left(1-\alpha_{n}\right) a_{n}+\sigma_{n}, \quad n \geq 1,
$$

where $\left\{\alpha_{n}\right\}$ is a sequence in $(0,1)$ and $\left\{\sigma_{n}\right\}$ is a sequence in $\mathbb{R}$ such that

(1) $\sum_{n=1}^{\infty} \alpha_{n}=\infty$

(2) $\lim \sup _{n \rightarrow \infty}\left(\sigma_{n} / \alpha_{n}\right) \leq 0$ or $\sum_{n=1}^{\infty}\left|\sigma_{n}\right|<\infty$.

Then $\lim _{n \rightarrow \infty} a_{n}=0$.

Lemma 2.2 ([47]). Let $\left\{x_{n}\right\}$ and $\left\{l_{n}\right\}$ be bounded sequences in a Banach space E and let $\left\{\beta_{n}\right\}$ be a sequence in $[0,1]$ with $0<\liminf _{n \rightarrow \infty} \beta_{n} \leq \limsup _{n \rightarrow \infty} \beta_{n}<1$. Suppose $x_{n+1}=\left(1-\beta_{n}\right) l_{n}+\beta_{n} x_{n}$ for all integers $n \geq 1$ and $\lim \sup _{n \rightarrow \infty}\left(\left\|l_{n+1}-l_{n}\right\|-\left\|x_{n+1}-x_{n}\right\|\right) \leq 0$. Then, $\lim _{n \rightarrow \infty}\left\|l_{n}-x_{n}\right\|=0$.

Lemma 2.3 ([42, Proposition 2.1]). Assume that $C$ is a closed convex subset of Hilbert space $H$, and let $T: C \rightarrow C$ be a self-mapping of $C$,

(i) if $T$ is a $k$-strictly pseudocontractive mapping, then $T$ satisfies the Lipscchitz condition

$$
\|T x-T y\| \leq \frac{1+\kappa}{1-\kappa}\|x-y\| \quad \forall x, y \in C .
$$

(ii) if $T$ is a $k$-strictly pseudocontractive mapping, then the mapping $I-T$ is demiclosed(at 0 ). That is, if $\left\{x_{n}\right\}$ is a sequence in $C$ such that $x_{n}-\tilde{x}$ and $(I-T) x_{n} \rightarrow 0$, then $(I-T) \tilde{x}=0$.

(iii) if $T$ is a $k$-strictly pseudocontractive mapping, then the fixed point set $F(T)$ of $T$ is closed and convex so that the projection $P_{F(T)}$ is well defined.

Lemma 2.4 ([25]). Assume $A$ is a strongly positive linear bounded operator on a Hilbert space $H$ with coefficient $\bar{\gamma}>0$ and $0<\rho \leq\|A\|^{-1}$. Then $\|I-\rho A\| \leq 1-\rho \bar{\gamma}$.

The following lemmas can be obtained from Acedo and $\mathrm{Xu}$ [35, Proposition 2.6] easily.

Lemma 2.5. Let $H$ be a Hilbert space, $C$ be a closed convex subset of $H$. For any integer $N \geq 1$, assume that, for each $1 \leq i \leq N, T_{i}: C \rightarrow H$ is a $k_{i}$-strictly pseudocontractive mapping for some $0 \leq k_{i}<1$. Assume that $\left\{\xi_{i}\right\}_{i=1}^{N}$ is a positive sequence such that $\sum_{i=1}^{N} \xi_{i}=1$. Then $\sum_{i=1}^{N} \xi_{i} T_{i}$ is a $k$-strictly pseudocontractive mapping with $k=\max \left\{k_{i}: 1 \leq i \leq N\right\}$. 
Lemma 2.6. Let $\left\{T_{i}\right\}_{i=1}^{N}$ and $\left\{\xi_{i}\right\}_{i=1}^{N}$ be as in Lemma 2.5. Suppose that $\left\{T_{i}\right\}_{i=1}^{N}$ has a common fixed point in $C$. Then $F\left(\sum_{i=1}^{N} \xi_{i} T_{i}\right)=\bigcap_{i=1}^{N} F\left(T_{i}\right)$.

For solving the mixed equilibrium problem, let us give the following assumptions for a bifunction $\Theta, \varphi$ and the set $C$ :

(A1) $\Theta(x, x)=0$ for all $x \in C$;

(A2) $\Theta$ is monotone, that is, $\Theta(x, y)+\Theta(y, x) \leq 0$ for all $x, y \in C$;

(A3) for each $x, y, z \in C, \lim _{t \rightarrow 0} \Theta(t z+(1-t) x, y) \leq \Theta(x, y)$;

(A4) for each $x \in C, y \mapsto \Theta(x, y)$ is convex and lower semicontinuous;

(B1) For each $x \in H$ and $r>0$, there exists a bounded subset $D_{x} \subset C$, and $y_{x} \in C$ such that for any $z \in C \backslash D_{x}$,

$$
\Theta(z, y)+\varphi\left(y_{x}\right)+\frac{1}{r}\left\langle y_{x}-z, z-x\right\rangle<\varphi(z)
$$

(B2) $C$ is a bounded set.

By similar argument as in [48, proof of Lemma 2.3], we have the following result.

Lemma 2.7. Let $C$ be a nonempty closed convex subset of $H$. Let $\Theta: C \times C \rightarrow \mathbb{R}$ be a bifunction satifies (A1)-(A4) and let $\varphi: C \rightarrow \mathbb{R} \cup\{+\infty\}$ be a proper lower semicontinuous and convex function. Assume that either (B1) or (B2) holds. For $r>0$ and $x \in H$, define a mapping $T_{r}: H \rightarrow C$ as follows:

$$
T_{r}(x)=\left\{z \in C: \Theta(z, y)+\varphi(y)+\frac{1}{r}\langle y-z, z-x\rangle \geq \varphi(z), \forall y \in C\right\}
$$

for all $x \in H$. Then, the following conditions hold:

(i) for each $x \in H, T_{r}(x) \neq \emptyset$;

(ii) $T_{r}$ is single-valued;

(iii) $T_{r}$ is firmly nonexpansive, that is, for any $x, y \in H,\left\|T_{r} x-T_{r} y\right\|^{2} \leq\left\langle T_{r} x-T_{r} y, x-y\right\rangle$;

(iv) $F\left(T_{r}\right)=\operatorname{MEP}(\Theta, \varphi)$;

(v) $\operatorname{MEP}(\Theta, \varphi)$ is closed and convex.

\section{Main Results}

In this section, we derive a strong convergence of an iterative algorithm which solves the problem of finding a common element of the set of solutions of a mixed equilibrium problem, the set of fixed points of a $k$-strictly pseudocontractive mapping of $C$ into itself and the set of the variational inequality for an $\alpha$-inverse-strongly monotone mapping of $C$ into $H$ in a Hilbert space.

Theorem 3.1. Let $C$ be a nonempty closed convex subset of a Hilbert space $H$. Let $\Theta$ be a bifunction from $C \times C$ to $\mathbb{R}$ satifies (A1)-(A4) and $\varphi: C \rightarrow \mathbb{R} \cup\{+\infty\}$ be a proper lower semicontinuous and 
convex function. Let $T$ be a $k$-strictly pseudocontractive mapping of $C$ into itself. Let $f$ be a contraction of $C$ into itself with coefficient $\beta \in(0,1), B$ an $\alpha$-inverse-strongly monotone mapping of $C$ into $H$ such that $\Omega:=F(T) \cap \operatorname{VI}(B, C) \cap \operatorname{MEP}(\Theta, \varphi) \neq \emptyset$. Let $A$ be a strongly bounded linear self-adjoint operator with coefficient $\bar{\gamma}>0$ and $0<\gamma<\bar{\gamma} / \beta$. Assume that either (B1) or (B2) holds. Given the sequences $\left\{\alpha_{n}\right\},\left\{\beta_{n}\right\},\left\{\delta_{n}\right\},\left\{\lambda_{n}\right\}$, and $\left\{r_{n}\right\}$ in $[0,1]$ satisfyies the following conditions

(D1) $\lim _{n \rightarrow \infty} \alpha_{n}=0, \sum_{n=1}^{\infty} \alpha_{n}=\infty$;

(D2) $0<\liminf _{n \rightarrow \infty} \delta_{n} \leq \limsup _{n \rightarrow \infty} \delta_{n}<1$;

(D3) $0 \leq k \leq \beta_{n}<\varepsilon<1$ for all $n \geq 0$, and $\lim _{n \rightarrow \infty}\left|\beta_{n+1}-\beta_{n}\right|=0$;

(D4) $\left\{\lambda_{n}\right\} \subset[a, b]$ for some $a, b$ with $0<a<b<2 \alpha$, and $\lim _{n \rightarrow \infty}\left|\lambda_{n+1}-\lambda_{n}\right|=0$;

(D5) $\liminf _{n \rightarrow \infty} r_{n}>0, \lim _{n \rightarrow \infty}\left|r_{n+1}-r_{n}\right|=0$.

Let $\left\{x_{n}\right\},\left\{u_{n}\right\}$, and $\left\{y_{n}\right\}$ be sequences generated by

$$
\begin{gathered}
x_{1}=x \in C \text { chosen arbitrarily, } \\
\Theta\left(u_{n}, y\right)+\varphi(y)-\varphi\left(u_{n}\right)+\frac{1}{r_{n}}\left\langle y-u_{n}, u_{n}-x_{n}\right\rangle \geq 0, \quad \forall y \in C, \\
y_{n}=\beta_{n} u_{n}+\left(1-\beta_{n}\right) T u_{n}, \\
x_{n+1}=\alpha_{n} \gamma f\left(x_{n}\right)+\delta_{n} x_{n}+\left(\left(1-\delta_{n}\right) I-\alpha_{n} A\right) P_{C}\left(y_{n}-\lambda_{n} B y_{n}\right), \quad n \geq 1 .
\end{gathered}
$$

Then $\left\{x_{n}\right\},\left\{u_{n}\right\}$, and $\left\{y_{n}\right\}$ converge strongly to a point $z \in \Omega$ which is the unique solution of the variational inequality

$$
\langle(A-\gamma f) z, z-x\rangle \leq 0, \quad \forall x \in \Omega
$$

Equivalently, one has $z=P_{\Omega}(I-A+\gamma f)(z)$.

Proof. Since $\lim _{n \rightarrow \infty} \alpha_{n}=0$, we may assume, without loss of generality, that $\alpha_{n}<\|A\|^{-1}$ for all $n$. By Lemma 2.4, we have $\left\|I-\alpha_{n} A\right\| \leq 1-\alpha_{n} \bar{\gamma}$. We will assume that $\|I-A\| \leq 1-\bar{\gamma}$. Observe that $P_{\Omega}(I-A+\gamma f)$ is a contraction. Indeed, for all $x, y \in C$, we have

$$
\begin{aligned}
\left\|P_{\Omega}(I-A+\gamma f)(x)-P_{\Omega}(I-A+\gamma f)(y)\right\| & \leq\|(I-A+\gamma f)(x)-(I-A+\gamma f)(y)\| \\
& \leq\|I-A\|\|x-y\|+\gamma\|f(x)-f(y)\| \\
& \leq(1-\bar{\gamma})\|x-y\|+\gamma \beta\|x-y\| \\
& =(1-(\bar{\gamma}-\gamma \beta))\|x-y\| .
\end{aligned}
$$

Since $H$ is complete, there exists a unique element $z \in C$ such that $z=P_{\Omega}(I-A+\gamma f)(z)$. On the other hand, since $A$ is a linear bounded self-adjoint operator, one has

$$
\|A\|=\sup \{|\langle A x, x\rangle|: x \in H,\|x\|=1\} .
$$


Observing that

$$
\begin{aligned}
\left\langle\left(\left(1-\delta_{n}\right) I-\alpha_{n} A\right) x, x\right\rangle & =1-\delta_{n}-\alpha_{n}\langle A x, x\rangle \\
& \geq 1-\delta_{n}-\alpha_{n}\|A\| \\
& \geq 0,
\end{aligned}
$$

we obtain $\left(1-\delta_{n}\right) I-\alpha_{n} A$ is positive. It follows that

$$
\begin{aligned}
\left\|\left(1-\delta_{n}\right) I-\alpha_{n} A\right\| & =\sup \left\{\left\langle\left(\left(1-\delta_{n}\right) I-\alpha_{n} A\right) x, x\right\rangle: x \in H,\|x\|=1\right\} \\
& =\sup \left\{1-\delta_{n}-\alpha_{n}\langle A x, x\rangle: x \in H,\|x\|=1\right\} \\
& \leq 1-\delta_{n}-\alpha_{n} \bar{\gamma} .
\end{aligned}
$$

Next, we divide the proof into six steps as follows.

Step 1. First we prove that $I-\lambda_{n} B$ is nonexpansive. For all $x, y \in C$ and $\lambda_{n} \in[0,2 \alpha]$,

$$
\begin{aligned}
\left\|\left(I-\lambda_{n} B\right) x-\left(I-\lambda_{n} B\right) y\right\|^{2} & =\left\|(x-y)-\lambda_{n}(B x-B y)\right\|^{2} \\
& =\|x-y\|^{2}-2 \lambda_{n}\langle x-y, B x-B y\rangle+\lambda_{n}^{2}\|B x-B y\|^{2} \\
& \leq\|x-y\|^{2}+\lambda_{n}\left(\lambda_{n}-2 \alpha\right)\|B x-B y\|^{2},
\end{aligned}
$$

which implies that $I-\lambda_{n} B$ is nonexpansive.

Step 2. Next we prove that $\left\{x_{n}\right\},\left\{y_{n}\right\},\left\{u_{n}\right\},\left\{B x_{n}\right\},\left\{B y_{n}\right\}$ and $\left\{B u_{n}\right\}$ are bounded. Indeed, pick any $p \in \Omega$. From (2.5), we have $p=P_{C}\left(p-\lambda_{n} B p\right)$. Setting $v_{n}=P_{C}\left(y_{n}-\lambda_{n} B y_{n}\right)$, we obtain from the nonexpansivity of $I-\lambda_{n} B$ that

$$
\begin{aligned}
\left\|v_{n}-p\right\| & =\left\|P_{C}\left(y_{n}-\lambda_{n} B y_{n}\right)-P_{C}\left(p-\lambda_{n} B p\right)\right\| \\
& \leq\left\|\left(y_{n}-\lambda_{n} B y_{n}\right)-\left(p-\lambda_{n} B p\right)\right\| \leq\left\|y_{n}-p\right\| .
\end{aligned}
$$

From (2.1), we have

$$
\begin{aligned}
\left\|y_{n}-p\right\|^{2} & =\left\|\beta_{n}\left(u_{n}-p\right)+\left(1-\beta_{n}\right)\left(T u_{n}-p\right)\right\|^{2} \\
& \leq \beta_{n}\left\|u_{n}-p\right\|^{2}-\left(1-\beta_{n}\right) \beta_{n}\left\|u_{n}-T u_{n}\right\|^{2}+\left(1-\beta_{n}\right)\left\|T u_{n}-p\right\|^{2}
\end{aligned}
$$

so, by (3.9) and the $k$-strict pseudocontractivity of $T$, it follows that

$$
\begin{aligned}
\left\|y_{n}-p\right\|^{2} & \leq\left\|u_{n}-p\right\|^{2}-\left(1-\beta_{n}\right)\left(\beta_{n}-k\right)\left\|u_{n}-T u_{n}\right\|^{2} \\
& \leq\left\|u_{n}-p\right\|^{2},
\end{aligned}
$$


that is,

$$
\left\|y_{n}-p\right\| \leq\left\|u_{n}-p\right\|
$$

Observe that

$$
\left\|u_{n}-p\right\|=\left\|T_{r_{n}} x_{n}-T_{r_{n}} p\right\| \leq\left\|x_{n}-p\right\| .
$$

From (3.8), (3.11) and the last inequality, we have

$$
\left\|v_{n}-p\right\| \leq\left\|x_{n}-p\right\| .
$$

It follows that

$$
\begin{aligned}
\left\|x_{n+1}-p\right\| & =\left\|\alpha_{n} \gamma f\left(x_{n}\right)+\delta_{n} x_{n}+\left(\left(1-\delta_{n}\right) I-\alpha_{n} A\right) v_{n}-p\right\| \\
& =\left\|\alpha_{n}\left(\gamma f\left(x_{n}\right)-A p\right)+\delta_{n}\left(x_{n}-p\right)+\left(\left(1-\delta_{n}\right) I-\alpha_{n} A\right)\left(v_{n}-p\right)\right\| \\
& \leq \alpha_{n}\left\|\gamma f\left(x_{n}\right)-A p\right\|+\delta_{n}\left\|x_{n}-p\right\|+\left(1-\delta_{n}-\alpha_{n} \bar{\gamma}\right)\left\|v_{n}-p\right\| \\
& \leq \alpha_{n} \gamma\left\|f\left(x_{n}\right)-f(p)\right\|+\alpha_{n}\|\gamma f(p)-A p\|+\left(1-\alpha_{n} \bar{\gamma}\right)\left\|x_{n}-p\right\| \\
& =\left[1-\alpha_{n}(\bar{\gamma}-\gamma \beta)\right]\left\|x_{n}-p\right\|+\alpha_{n}\|\gamma f(p)-A p\| \\
& =\left[1-\alpha_{n}(\bar{\gamma}-\gamma \beta)\right]\left\|x_{n}-p\right\|+\alpha_{n}(\bar{\gamma}-\gamma \beta) \frac{\|\gamma f(p)-A p\|}{\bar{\gamma}-\gamma \beta} .
\end{aligned}
$$

By simple induction, we have

$$
\left\|x_{n}-p\right\| \leq \max \left\{\left\|x_{1}-p\right\|, \frac{\|A p-\gamma f(p)\|}{\bar{\gamma}-\gamma \beta}\right\},
$$

which gives that the sequence $\left\{x_{n}\right\}$ is bounded, so are $\left\{y_{n}\right\},\left\{u_{n}\right\},\left\{B x_{n}\right\},\left\{B y_{n}\right\}$, and $\left\{B u_{n}\right\}$. Step 3. Next we claim that

$$
\lim _{n \rightarrow \infty}\left\|x_{n+1}-x_{n}\right\|=0
$$

Notice that

$$
\begin{aligned}
\left\|v_{n}-v_{n-1}\right\| & =\left\|P_{C}\left(y_{n}-\lambda_{n} B y_{n}\right)-P_{C}\left(y_{n-1}-\lambda_{n-1} B y_{n-1}\right)\right\| \\
& \leq\left\|\left(y_{n}-\lambda_{n} B y_{n}\right)-\left(y_{n-1}-\lambda_{n-1} B y_{n-1}\right)\right\| \\
& =\left\|\left(y_{n}-\lambda_{n} B y_{n}\right)-\left(y_{n-1}-\lambda_{n} B y_{n-1}\right)+\left(\lambda_{n-1}-\lambda_{n}\right) B y_{n-1}\right\| \\
& \leq\left\|\left(y_{n}-\lambda_{n} B y_{n}\right)-\left(y_{n-1}-\lambda_{n} B y_{n-1}\right)\right\|+\left|\lambda_{n-1}-\lambda_{n}\right|\left\|B y_{n-1}\right\| \\
& \leq\left\|y_{n}-y_{n-1}\right\|+\left|\lambda_{n-1}-\lambda_{n}\right|\left\|B y_{n-1}\right\| .
\end{aligned}
$$


Next, we define

$$
V_{n}=\left(1-\beta_{n}\right) T+\beta_{n} I
$$

As shown in [19], from the $k$-strict pseudocontractivity of $T$ and the conditions (D4), it follows that $V_{n}$ is a nonexpansive maping for which $F(T)=F\left(V_{n}\right)$.

Observing that

$$
\begin{gathered}
y_{n}=V_{n} u_{n}, \\
y_{n-1}=V_{n-1} u_{n-1},
\end{gathered}
$$

we have

$$
\begin{aligned}
\left\|y_{n}-y_{n-1}\right\| & =\left\|V_{n} u_{n}-V_{n-1} u_{n-1}\right\| \\
& \leq\left\|V_{n} u_{n}-V_{n} u_{n-1}\right\|+\left\|V_{n} u_{n-1}-V_{n-1} u_{n-1}\right\| \\
& \leq\left\|u_{n}-u_{n-1}\right\|+\left\|V_{n} u_{n-1}-V_{n-1} u_{n-1}\right\| \\
& =\left\|u_{n}-u_{n-1}\right\|+\left\|\left(\beta_{n} u_{n-1}+\left(1-\beta_{n}\right) T u_{n-1}\right)-\left(\beta_{n-1} u_{n-1}+\left(1-\beta_{n-1}\right) T u_{n-1}\right)\right\| \\
& \leq\left\|u_{n}-u_{n-1}\right\|+M_{1}\left|\beta_{n}-\beta_{n-1}\right|
\end{aligned}
$$

where $M_{1}$ is an appropriate constant such that $M_{1} \geq \sup _{n \geq 1}\left\{\left\|u_{n}\right\|,\left\|T u_{n}\right\|\right\}$. Substituting (3.20) into (3.17), we obtain

$$
\begin{aligned}
\left\|v_{n}-v_{n-1}\right\| & \leq\left\|y_{n}-y_{n-1}\right\|+\left|\lambda_{n-1}-\lambda_{n}\right|\left\|B y_{n-1}\right\| \\
& \leq\left\|u_{n}-u_{n-1}\right\|+M_{1}\left|\beta_{n}-\beta_{n-1}\right|+\left|\lambda_{n-1}-\lambda_{n}\right|\left\|B y_{n-1}\right\|
\end{aligned}
$$

On the other hand, from $u_{n}=T_{r_{n}} x_{n} \in \operatorname{dom} \varphi$ and $u_{n+1}=T_{r_{n+1}} x_{n+1} \in \operatorname{dom} \varphi$, we note that

$$
\begin{aligned}
\Theta\left(u_{n}, y\right)+\varphi(y)-\varphi\left(u_{n}\right)+\frac{1}{r_{n}}\left\langle y-u_{n}, u_{n}-x_{n}\right\rangle \geq 0 \quad \forall y \in C, \\
\Theta\left(u_{n+1}, y\right)+\varphi(y)-\varphi\left(u_{n+1}\right)+\frac{1}{r_{n+1}}\left\langle y-u_{n+1}, u_{n+1}-x_{n+1}\right\rangle \geq 0 \quad \forall y \in C .
\end{aligned}
$$

Putting $y=u_{n+1}$ in (3.22) and $y=u_{n}$ in (3.23), we have

$$
\begin{gathered}
\Theta\left(u_{n}, u_{n+1}\right)+\varphi\left(u_{n+1}\right)-\varphi\left(u_{n}\right)+\frac{1}{r_{n}}\left\langle u_{n+1}-u_{n}, u_{n}-x_{n}\right\rangle \geq 0 \\
\Theta\left(u_{n+1}, u_{n}\right)+\varphi\left(u_{n}\right)-\varphi\left(u_{n+1}\right)+\frac{1}{r_{n+1}}\left\langle u_{n}-u_{n+1}, u_{n+1}-x_{n+1}\right\rangle \geq 0
\end{gathered}
$$


So, from (A2) we have

$$
\left\langle u_{n+1}-u_{n}, \frac{u_{n}-x_{n}}{r_{n}}-\frac{u_{n+1}-x_{n+1}}{r_{n+1}}\right\rangle \geq 0,
$$

and hence

$$
\left\langle u_{n+1}-u_{n}, u_{n}-u_{n+1}+u_{n+1}-x_{n}-\frac{r_{n}}{r_{n+1}}\left(u_{n+1}-x_{n+1}\right)\right\rangle \geq 0 .
$$

Without loss of generality, let us assume that there exists a real number $c$ such that $r_{n}>c>0$ for all $n \in \mathbb{N}$. Then, we have

$$
\begin{aligned}
\left\|u_{n+1}-u_{n}\right\|^{2} & \leq\left\langle u_{n+1}-u_{n}, x_{n+1}-x_{n}+\left(1-\frac{r_{n}}{r_{n+1}}\right)\left(u_{n+1}-x_{n+1}\right)\right\rangle \\
& \leq\left\|u_{n+1}-u_{n}\right\|\left\{\left\|x_{n+1}-x_{n}\right\|+\left|1-\frac{r_{n}}{r_{n+1}}\right|\left\|u_{n+1}-x_{n+1}\right\|\right\},
\end{aligned}
$$

and hence

$$
\begin{aligned}
\left\|u_{n+1}-u_{n}\right\| & \leq\left\|x_{n+1}-x_{n}\right\|+\frac{1}{r_{n+1}}\left|r_{n+1}-r_{n}\right|\left\|u_{n+1}-x_{n+1}\right\| \\
& \leq\left\|x_{n+1}-x_{n}\right\|+\frac{1}{c}\left|r_{n+1}-r_{n}\right| M_{2}
\end{aligned}
$$

where $M_{2}=\sup \left\{\left\|u_{n}-x_{n}\right\|: n \in \mathbb{N}\right\}$. It follows from (3.21) and the last inequality that

$$
\left\|v_{n}-v_{n-1}\right\| \leq\left\|x_{n+1}-x_{n}\right\|+M\left(\frac{1}{c}\left|r_{n+1}-r_{n}\right|+\left|\beta_{n}-\beta_{n-1}\right|\right)+\left|\lambda_{n-1}-\lambda_{n}\right|\left\|B y_{n-1}\right\|,
$$

where $M=\max \left\{M_{1}, M_{2}\right\}$.

Define a sequence $\left\{l_{n}\right\}$ such that

$$
x_{n+1}=\left(1-\delta_{n}\right) l_{n}+\delta_{n} x_{n}, \quad \forall n \geq 1 .
$$


Then, we have

$$
\begin{aligned}
l_{n+1}-l_{n}= & \frac{x_{n+2}-\delta_{n+1} x_{n+1}}{1-\delta_{n+1}}-\frac{x_{n+1}-\delta_{n} x_{n}}{1-\delta_{n}} \\
= & \frac{\alpha_{n+1} \gamma f\left(x_{n+1}\right)+\left(\left(1-\delta_{n+1}\right) I-\alpha_{n+1} A\right) v_{n+1}}{1-\delta_{n+1}} \\
& -\frac{\alpha_{n} \gamma f\left(x_{n}\right)+\left(\left(1-\delta_{n}\right) I-\alpha_{n} A\right) v_{n}}{1-\delta_{n}} \\
= & \frac{\alpha_{n+1}}{1-\delta_{n+1}}\left(\gamma f\left(x_{n+1}\right)-A v_{n+1}\right)+\frac{\alpha_{n}}{1-\delta_{n}}\left(A v_{n}-\gamma f\left(x_{n}\right)\right) \\
& +v_{n+1}-v_{n} .
\end{aligned}
$$

It follows from (3.29) that

$$
\begin{aligned}
\left\|l_{n+1}-l_{n}\right\|-\left\|x_{n}-x_{n+1}\right\| \leq & \frac{\alpha_{n+1}}{1-\delta_{n+1}}\left\|\gamma f\left(x_{n+1}\right)-A v_{n+1}\right\| \\
& +\frac{\alpha_{n}}{1-\delta_{n}}\left\|A v_{n}-\gamma f\left(x_{n}\right)\right\|+\left\|v_{n+1}-v_{n}\right\|-\left\|x_{n}-x_{n+1}\right\| \\
\leq & \frac{\alpha_{n+1}}{1-\delta_{n+1}}\left\|\gamma f\left(x_{n+1}\right)-A v_{n+1}\right\|+\frac{\alpha_{n}}{1-\delta_{n}}\left\|A v_{n}-\gamma f\left(x_{n}\right)\right\| \\
& +M\left(\frac{1}{c}\left|r_{n+1}-r_{n}\right|+\left|\beta_{n}-\beta_{n-1}\right|\right)+\left|\lambda_{n-1}-\lambda_{n}\right|\left\|B y_{n-1}\right\| .
\end{aligned}
$$

Observing the conditions (D1), (D3), (D4), (D5), and taking the superior limit as $n \rightarrow \infty$, we get

$$
\limsup _{n \rightarrow \infty}\left(\left\|l_{n+1}-l_{n}\right\|-\left\|x_{n}-x_{n+1}\right\|\right) \leq 0
$$

We can obtain $\lim _{n \rightarrow \infty}\left\|l_{n}-x_{n}\right\|=0$ easily by Lemma 2.2 . Observing that

$$
x_{n+1}-x_{n}=\left(1-\delta_{n}\right)\left(l_{n}-x_{n}\right),
$$

we obtain

$$
\lim _{n \rightarrow \infty}\left\|x_{n+1}-x_{n}\right\|=0
$$

Hence (3.16) is proved.

Step 4. Next we prove that

$$
\lim _{n \rightarrow \infty}\left\|T v_{n}-v_{n}\right\|=0 .
$$


Fixed Point Theory and Applications

(a) First we prove that $\lim _{n \rightarrow \infty}\left\|x_{n}-v_{n}\right\|=0$. Observing that

$$
\begin{aligned}
x_{n}-v_{\mathrm{n}} & =x_{n}-x_{n+1}+x_{n+1}-v_{n} \\
& =x_{n}-x_{n+1}+\alpha_{n} \gamma f\left(x_{n}\right)+\delta_{n} x_{n}+\left(\left(1-\delta_{n}\right) I-\alpha_{n} A\right) v_{n}-v_{n} \\
& =x_{n}-x_{n+1}+\alpha_{n}\left(\gamma f\left(x_{n}\right)-A v_{n}\right)+\delta_{n}\left(x_{n}-v_{n}\right),
\end{aligned}
$$

we arrive at

$$
\left(1-\delta_{n}\right)\left(x_{n}-v_{n}\right)=x_{n}-x_{n+1}+\alpha_{n}\left(\gamma f\left(x_{n}\right)-A v_{n}\right),
$$

which implies that

$$
\left(1-\delta_{n}\right)\left\|x_{n}-v_{n}\right\| \leq\left\|x_{n}-x_{n+1}\right\|+\alpha_{n}\left\|\gamma f\left(x_{n}\right)-A v_{n}\right\| .
$$

Therefore, it follows from (3.16), (D1), and (D2) that

$$
\lim _{n \rightarrow \infty}\left\|x_{n}-v_{n}\right\|=0
$$

(b) Next, we will show that $\lim _{n \rightarrow \infty}\left\|B y_{n}-B p\right\|=0$ for any $p \in \Omega$. Observe that

$$
\begin{aligned}
\left\|x_{n+1}-p\right\|^{2}= & \left\|\left(\left(1-\delta_{\mathrm{n}}\right) I-\alpha_{n} A\right)\left(v_{n}-p\right)+\delta_{n}\left(x_{n}-p\right)+\alpha_{n}\left(\gamma f\left(x_{n}\right)-A p\right)\right\|^{2} \\
= & \left\|\left(\left(1-\delta_{n}\right) I-\alpha_{n} A\right)\left(v_{n}-p\right)+\delta_{n}\left(x_{n}-p\right)\right\|^{2}+\alpha_{n}^{2}\left\|\gamma f\left(x_{n}\right)-A p\right\|^{2} \\
& +2 \delta_{n} \alpha_{n}\left\langle x_{n}-p, \gamma f\left(x_{n}\right)-A p\right\rangle+2 \alpha_{n}\left\langle\left(\left(1-\delta_{n}\right) I-\alpha_{n} A\right)\left(v_{n}-p\right), \gamma f\left(x_{n}\right)-A p\right\rangle \\
\leq & \left(\left(1-\delta_{n}-\alpha_{n} \bar{\gamma}\right)\left\|v_{n}-p\right\|+\delta_{n}\left\|x_{n}-p\right\|\right)^{2}+\alpha_{n}^{2}\left\|\gamma f\left(x_{n}\right)-A p\right\|^{2} \\
& +2 \delta_{n} \alpha_{n}\left\langle x_{n}-p, \gamma f\left(x_{n}\right)-A p\right\rangle+2 \alpha_{n}\left\langle\left(\left(1-\delta_{n}\right) I-\alpha_{n} A\right)\left(v_{n}-p\right), \gamma f\left(x_{n}\right)-A p\right\rangle \\
= & \left(1-\delta_{n}-\alpha_{n} \bar{\gamma}\right)^{2}\left\|v_{n}-p\right\|^{2}+\delta_{n}^{2}\left\|x_{n}-p\right\|^{2} \\
& +2\left(1-\delta_{n}-\alpha_{n} \bar{\gamma}\right) \delta_{n}\left\|v_{n}-p\right\|\left\|x_{n}-p\right\|+c_{n} \\
\leq & \left(1-\delta_{n}-\alpha_{n} \bar{\gamma}\right)^{2}\left\|v_{n}-p\right\|^{2}+\delta_{n}^{2}\left\|x_{n}-p\right\|^{2} \\
& +\left(1-\delta_{n}-\alpha_{n} \bar{\gamma}\right) \delta_{n}\left(\left\|v_{n}-p\right\|^{2}+\left\|x_{n}-p\right\|^{2}\right)+c_{n} \\
= & \left.\left(1-\alpha_{n} \bar{\gamma}\right)^{2}-2\left(1-\alpha_{n} \bar{\gamma}\right) \delta_{n}+\delta_{n}^{2}\right]\left\|v_{n}-p\right\|^{2}+\delta_{n}^{2}\left\|x_{n}-p\right\|^{2} \\
& +\left(\left(1-\alpha_{n} \bar{\gamma}\right) \delta_{n}-\delta_{n}^{2}\right)\left(\left\|v_{n}-p\right\|^{2}+\left\|x_{n}-p\right\|^{2}\right)+c_{n}
\end{aligned}
$$




$$
\begin{aligned}
= & \left(1-\alpha_{n} \bar{\gamma}\right)^{2}\left\|v_{n}-p\right\|^{2}-\left(1-\alpha_{n} \bar{\gamma}\right) \delta_{n}\left\|v_{n}-p\right\|^{2}+\left(1-\alpha_{n} \bar{\gamma}\right) \delta_{n}\left\|x_{n}-p\right\|^{2}+c_{n} \\
= & \left(1-\alpha_{n} \bar{\gamma}\right)\left(1-\delta_{n}-\alpha_{n} \bar{\gamma}\right)\left\|v_{n}-p\right\|^{2}+\left(1-\alpha_{n} \bar{\gamma}\right) \delta_{n}\left\|x_{n}-p\right\|^{2}+c_{n} \\
\leq & \left(1-\alpha_{n} \bar{\gamma}\right)\left(1-\delta_{n}-\alpha_{n} \bar{\gamma}\right)\left[\left\|\left(y_{n}-\lambda_{n} B y_{n}\right)-\left(p-\lambda_{n} B p\right)\right\|^{2}\right] \\
& +\left(1-\alpha_{n} \bar{\gamma}\right) \delta_{n}\left\|x_{n}-p\right\|^{2}+c_{n} \\
\leq & \left(1-\alpha_{n} \bar{\gamma}\right)\left(1-\delta_{n}-\alpha_{n} \bar{\gamma}\right)\left[\left\|y_{n}-p\right\|^{2}+\lambda_{n}\left(\lambda_{n}-2 \alpha\right)\left\|B y_{n}-B p\right\|^{2}\right] \\
& +\left(1-\alpha_{n} \bar{\gamma}\right) \delta_{n}\left\|x_{n}-p\right\|^{2}+c_{n} \\
\leq & \left\|x_{n}-p\right\|^{2}+b(b-2 \alpha)\left\|B y_{n}-B p\right\|^{2}+c_{n},
\end{aligned}
$$

where

$$
\begin{aligned}
c_{n}= & \alpha_{n}^{2}\left\|\gamma f\left(x_{n}\right)-A p\right\|^{2}+2 \delta_{n} \alpha_{n}\left\|x_{n}-p\right\|\left\|\gamma f\left(x_{n}\right)-A p\right\| \\
& +2 \alpha_{n}\left\|\left(\left(1-\delta_{n}\right) I-\alpha_{n} A\right)\left(v_{n}-p\right)\right\|\left\|\gamma f\left(x_{n}\right)-A p\right\| .
\end{aligned}
$$

This implies that

$$
\begin{aligned}
-b(b-2 \alpha)\left\|B y_{n}-B p\right\|^{2} & \leq\left\|x_{n}-p\right\|^{2}-\left\|x_{n+1}-p\right\|^{2}+c_{n} \\
& \leq\left\|x_{n}-x_{n+1}\right\|\left(\left\|x_{n}-p\right\|+\left\|x_{n+1}-p\right\|\right)+c_{n} .
\end{aligned}
$$

It is easy to see that $\lim _{n \rightarrow \infty} c_{n}=0$ and then from (3.16), we obtain

$$
\lim _{n \rightarrow \infty}\left\|B y_{n}-B p\right\|=0
$$

(c) Next we prove that $\lim _{n \rightarrow \infty}\left\|x_{n}-u_{n}\right\|=0$. From (2.3), we have

$$
\begin{aligned}
\left\|v_{n}-p\right\|^{2}= & \left\|P_{C}\left(y_{n}-\lambda_{n} B y_{n}\right)-P_{C}\left(p-\lambda_{n} B p\right)\right\|^{2} \\
\leq & \left\langle\left(y_{n}-\lambda_{n} B y_{n}\right)-\left(p-\lambda_{n} B p\right), v_{n}-p\right\rangle \\
= & \frac{1}{2}\left\{\left\|\left(y_{n}-\lambda_{n} B y_{n}\right)-\left(p-\lambda_{n} B p\right)\right\|^{2}+\left\|v_{n}-p\right\|^{2}\right. \\
& \left.\quad-\left\|\left(y_{n}-\lambda_{n} B y_{n}\right)-\left(p-\lambda_{n} B p\right)-\left(v_{n}-p\right)\right\|^{2}\right\} \\
\leq & \frac{1}{2}\left\{\left\|y_{n}-p\right\|^{2}+\left\|v_{n}-p\right\|^{2}-\left\|\left(y_{n}-v_{n}\right)-\lambda_{n}\left(B y_{n}-B p\right)\right\|^{2}\right\} \\
= & \frac{1}{2}\left\{\left\|y_{n}-p\right\|^{2}+\left\|v_{n}-p\right\|^{2}-\left\|y_{n}-v_{n}\right\|^{2}+2 \lambda_{n}\left\langle y_{n}-v_{n}, B y_{n}-B p\right\rangle-\lambda_{n}^{2}\left\|B y_{n}-B p\right\|^{2}\right\},
\end{aligned}
$$


so, we obtain

$$
\left\|v_{n}-p\right\|^{2} \leq\left\|y_{n}-p\right\|^{2}-\left\|y_{n}-v_{n}\right\|^{2}+2 \lambda_{n}\left\langle y_{n}-v_{n}, B y_{n}-B p\right\rangle-\lambda_{n}^{2}\left\|B y_{n}-B p\right\|^{2} .
$$

It follows that

$$
\begin{aligned}
\left\|x_{n+1}-p\right\|^{2} \leq & \left(1-\alpha_{n} \bar{\gamma}\right)\left(1-\delta_{n}-\alpha_{n} \bar{\gamma}\right)\left\|v_{n}-p\right\|^{2}+\left(1-\alpha_{n} \bar{\gamma}\right) \delta_{n}\left\|x_{n}-p\right\|^{2}+c_{n} \\
\leq & \left(1-\alpha_{n} \bar{\gamma}\right)\left(1-\delta_{n}-\alpha_{n} \bar{\gamma}\right) \\
& \times\left[\left\|y_{n}-p\right\|^{2}-\left\|y_{n}-v_{n}\right\|^{2}+2 \lambda_{n}\left\langle y_{n}-v_{n}, B y_{n}-B p\right\rangle-\lambda_{n}^{2}\left\|B y_{n}-B p\right\|^{2}\right] \\
& +\left(1-\alpha_{n} \bar{\gamma}\right) \delta_{n}\left\|x_{n}-p\right\|^{2}+c_{n} \\
\leq & \left(1-\alpha_{n} \bar{\gamma}\right)\left\|x_{n}-p\right\|^{2}-\left(1-\alpha_{n} \bar{\gamma}\right)\left(1-\delta_{n}-\alpha_{n} \bar{\gamma}\right)\left\|y_{n}-v_{n}\right\|^{2} \\
& +2 \lambda_{n}\left(1-\alpha_{n} \bar{\gamma}\right)\left(1-\delta_{n}-\alpha_{n} \bar{\gamma}\right)\left\|y_{n}-v_{n}\right\|\left\|B y_{n}-B p\right\| \\
& -\lambda_{n}^{2}\left(1-\alpha_{n} \bar{\gamma}\right)\left(1-\delta_{n}-\alpha_{n} \bar{\gamma}\right)\left\|B y_{n}-B p\right\|^{2}+c_{n},
\end{aligned}
$$

which implies that

$$
\begin{aligned}
\left(1-\alpha_{n} \bar{\gamma}\right)\left(1-\delta_{n}-\alpha_{n} \bar{\gamma}\right)\left\|y_{n}-v_{n}\right\|^{2} \leq & \left\|x_{n}-p\right\|^{2}-\left\|x_{n+1}-p\right\|^{2} \\
& +2 \lambda_{n}\left(1-\alpha_{n} \bar{\gamma}\right)\left(1-\delta_{n}-\alpha_{n} \bar{\gamma}\right)\left\|y_{n}-v_{n}\right\|\left\|B y_{n}-B p\right\| \\
& -\lambda_{n}^{2}\left(1-\alpha_{n} \bar{\gamma}\right)\left(1-\delta_{n}-\alpha_{n} \bar{\gamma}\right)\left\|B y_{n}-B p\right\|^{2}+c_{n} \\
\leq & \left\|x_{n}-x_{n+1}\right\|\left(\left\|x_{n}-p\right\|+\left\|x_{n+1}-p\right\|\right) \\
& +2 \lambda_{n}\left(1-\alpha_{n} \bar{\gamma}\right)\left(1-\delta_{n}-\alpha_{n} \bar{\gamma}\right)\left\|y_{n}-v_{n}\right\|\left\|B y_{n}-B p\right\| \\
& -\lambda_{n}^{2}\left(1-\alpha_{n} \bar{\gamma}\right)\left(1-\delta_{n}-\alpha_{n} \bar{\gamma}\right)\left\|B y_{n}-B p\right\|^{2}+c_{n} .
\end{aligned}
$$

Applying (3.16), (3.44), $\limsup _{n \rightarrow \infty} \delta_{n}<1$, and $\lim _{n \rightarrow \infty} c_{n}=0$ to the last inequality, we obtain that

$$
\lim _{n \rightarrow \infty}\left\|y_{n}-v_{n}\right\|=0
$$

It follows from (3.40) and (3.49) that

$$
\left\|x_{n}-y_{n}\right\| \leq\left\|x_{n}-v_{n}\right\|+\left\|v_{n}-y_{n}\right\| \longrightarrow 0 \text { as } n \longrightarrow \infty .
$$

Then it follows from (D1), (3.49) and (3.50) that

$$
\begin{aligned}
\left\|x_{n+1}-y_{n}\right\| & =\left\|\alpha_{n}\left(\gamma f\left(x_{n}\right)-A y_{n}\right)+\delta_{n}\left(x_{n}-y_{n}\right)+\left(\left(1-\delta_{n}\right) I-\alpha_{n} A\right)\left(v_{n}-y_{n}\right)\right\| \\
& \leq \alpha_{n}\left\|\gamma f\left(x_{n}\right)-A y_{n}\right\|+\delta_{n}\left\|x_{n}-y_{n}\right\|+\left(1-\delta_{n}-\alpha_{n} \bar{\gamma}\right)\left\|v_{n}-y_{n}\right\| \longrightarrow 0 .
\end{aligned}
$$


For any $p \in \Omega$, we have from Lemma 2.7,

$$
\begin{aligned}
\left\|u_{n}-p\right\|^{2} & =\left\|T_{r_{n}} x_{n}-T_{r_{n}} p\right\|^{2} \leq\left\langle T_{r_{n}} x_{n}-T_{r_{n}} p, x_{n}-p\right\rangle \\
& =\left\langle u_{n}-p, x_{n}-p\right\rangle=\frac{1}{2}\left(\left\|u_{n}-p\right\|^{2}+\left\|x_{n}-p\right\|^{2}-\left\|x_{n}-u_{n}\right\|^{2}\right) .
\end{aligned}
$$

Hence

$$
\left\|u_{n}-p\right\|^{2} \leq\left\|x_{n}-p\right\|^{2}-\left\|x_{n}-u_{n}\right\|^{2} .
$$

From (3.41) we observe that

$$
\begin{aligned}
\left\|x_{n+1}-p\right\|^{2} \leq & \left(1-\delta_{n}-\alpha_{n} \bar{\gamma}\right)^{2}\left\|v_{n}-p\right\|^{2}+\delta_{n}^{2}\left\|x_{n}-p\right\|^{2} \\
& +2\left(1-\delta_{n}-\alpha_{n} \bar{\gamma}\right) \delta_{n}\left\|v_{n}-p\right\|\left\|x_{n}-p\right\|+c_{n} \\
\leq & \left(1-\delta_{n}-\alpha_{n} \bar{\gamma}\right)^{2}\left\|u_{n}-p\right\|^{2}+\delta_{n}^{2}\left\|x_{n}-p\right\|^{2} \\
& +2\left(1-\delta_{n}-\alpha_{n} \bar{\gamma}\right) \delta_{n}\left\|u_{n}-p\right\| x_{n}-p \|+c_{n} \\
\leq & \left(1-\delta_{n}-\alpha_{n} \bar{\gamma}\right)^{2}\left\|u_{n}-p\right\|^{2}+\delta_{n}^{2}\left\|x_{n}-p\right\|^{2} \\
& +\left(1-\delta_{n}-\alpha_{n} \bar{\gamma}\right) \delta_{n}\left(\left\|u_{n}-p\right\|^{2}+\left\|x_{n}-p\right\|^{2}\right)+c_{n} \\
= & \left(\left(1-\alpha_{n} \bar{\gamma}\right)^{2}-2 \delta_{n}\left(1-\alpha_{n} \bar{\gamma}\right)+\delta_{n}^{2}\right)\left\|u_{n}-p\right\|^{2}+\delta_{n}^{2}\left\|x_{n}-p\right\|^{2} \\
& +\left(1-\alpha_{n} \bar{\gamma}\right) \delta_{n}\left(\left\|u_{n}-p\right\|^{2}+\left\|x_{n}-p\right\|^{2}\right)-\delta_{n}^{2}\left(\left\|u_{n}-p\right\|^{2}+\left\|x_{n}-p\right\|^{2}\right)+c_{n} \\
= & \left(\left(1-\alpha_{n} \bar{\gamma}\right)^{2}-2 \delta_{n}\left(1-\alpha_{n} \bar{\gamma}\right)+\delta_{n}^{2}+\left(1-\alpha_{n} \bar{\gamma}\right) \delta_{n}-\delta_{n}^{2}\right)\left\|u_{n}-p\right\|^{2}+\delta_{n}^{2}\left\|x_{n}-p\right\|^{2} \\
& +\left(1-\alpha_{n} \bar{\gamma}\right) \delta_{n}\left\|x_{n}-p\right\|^{2}-\delta_{n}^{2}\left\|x_{n}-p\right\|^{2}+c_{n} \\
= & \left(\left(1-\alpha_{n} \bar{\gamma}\right)^{2}-\delta_{n}\left(1-\alpha_{n} \bar{\gamma}\right)\right)\left\|u_{n}-p\right\|^{2}+\left(1-\alpha_{n} \bar{\gamma}\right) \delta_{n}\left\|x_{n}-p\right\|^{2}+c_{n} \\
\leq & \left(1-\alpha_{n} \bar{\gamma}\right)\left(1-\alpha_{n} \bar{\gamma}-\delta_{n}\right)\left(\left\|x_{n}-p\right\|^{2}-\left\|x_{n}-u_{n}\right\|^{2}\right)+\left(1-\alpha_{n} \bar{\gamma}\right) \delta_{n}\left\|x_{n}-p\right\|^{2}+c_{n} \\
= & \left(1-\alpha_{n} \bar{\gamma}\right)^{2}\left\|x_{n}-p\right\|^{2}-\left(1-\alpha_{n} \bar{\gamma}\right)\left(1-\alpha_{n} \bar{\gamma}-\delta_{n}\right)\left\|x_{n}-u_{n}\right\|^{2}+c_{n} \\
= & \left(1-2 \alpha_{n} \bar{\gamma}+\left(\alpha_{n} \bar{\gamma}\right)^{2}\right)\left\|x_{n}-p\right\|^{2}-\left(1-\alpha_{n} \bar{\gamma}\right)\left(1-\alpha_{n} \bar{\gamma}-\delta_{n}\right)\left\|x_{n}-u_{n}\right\|^{2}+c_{n} \\
\leq & \left\|x_{n}-p\right\|^{2}+\left(\alpha_{n} \bar{\gamma}\right)^{2}\left\|x_{n}-p\right\|^{2}-\left(1-\alpha_{n} \bar{\gamma}\right)\left(1-\alpha_{n} \bar{\gamma}-\delta_{n}\right)\left\|x_{n}-u_{n}\right\|^{2}+c_{n} . \\
& (3.54)
\end{aligned}
$$


Hence

$$
\begin{aligned}
\left(1-\alpha_{n} \bar{\gamma}\right)\left(1-\alpha_{n} \bar{\gamma}-\delta_{n}\right)\left\|x_{n}-u_{n}\right\|^{2} \leq & \left\|x_{n}-p\right\|^{2}-\left\|x_{n+1}-p\right\|^{2}+\left(\alpha_{n} \bar{\gamma}\right)^{2}\left\|x_{n}-p\right\|^{2}+c_{n} \\
= & \left(\left\|x_{n}-p\right\|-\left\|x_{n+1}-p\right\|\right)\left(\left\|x_{n}-p\right\|+\left\|x_{n+1}-p\right\|\right) \\
& +\left(\alpha_{n} \bar{\gamma}\right)^{2}\left\|x_{n}-p\right\|^{2}+c_{n} \\
\leq & \left\|x_{n}-x_{n+1}\right\|\left(\left\|x_{n}-p\right\|+\left\|x_{n+1}-p\right\|\right) \\
& +\left(\alpha_{n} \bar{\gamma}\right)^{2}\left\|x_{n}-p\right\|^{2}+c_{n} .
\end{aligned}
$$

Using (D1), (D2) and (3.16), we obtain

$$
\lim _{n \rightarrow \infty}\left\|u_{n}-x_{n}\right\|=0
$$

(d) Next we prove that $\lim _{n \rightarrow \infty}\left\|x_{n}-T x_{n}\right\|=0$. Using Lemma 2.3 (i), we have

$$
\begin{aligned}
\left\|T x_{n}-x_{n}\right\| \leq & \left\|x_{n}-x_{n+1}\right\|+\left\|x_{n+1}-y_{n}\right\|+\left\|y_{n}-T x_{n}\right\| \\
& \leq\left\|x_{n}-x_{n+1}\right\|+\left\|x_{n+1}-y_{n}\right\|+\beta_{n}\left\|u_{n}-T x_{n}\right\|+\left(1-\beta_{n}\right)\left\|T u_{n}-T x_{n}\right\| \\
& \leq\left\|x_{n}-x_{n+1}\right\|+\left\|x_{n+1}-y_{n}\right\|+\beta_{n}\left\|u_{n}-x_{n}\right\|+\beta_{n}\left\|x_{n}-T x_{n}\right\| \\
& +\left(1-\beta_{n}\right) \frac{1+k}{1-k}\left\|u_{n}-x_{n}\right\|
\end{aligned}
$$

which implies that

$$
\begin{aligned}
\left(1-\beta_{n}\right)\left\|T x_{n}-x_{n}\right\| \leq & \left\|x_{n}-x_{n+1}\right\|+\left\|x_{n+1}-y_{n}\right\| \\
& +\left(\frac{1+k}{1-k}+\beta_{n}\left(1-\frac{1+k}{1-k}\right)\right)\left\|u_{n}-x_{n}\right\| \longrightarrow 0 \quad \text { as } n \longrightarrow \infty .
\end{aligned}
$$

By (3.16), (3.51), and (3.56), we have

$$
\lim _{n \rightarrow \infty}\left\|T x_{n}-x_{n}\right\|=0
$$

Observing that

$$
\begin{aligned}
\left\|x_{n+1}-v_{n}\right\| & \leq\left\|\alpha_{n}\left(\gamma f\left(x_{n}\right)-A v_{n}\right)+\delta_{n}\left(x_{n}-v_{n}\right)\right\| \\
& \leq \alpha_{n}\left\|\gamma f\left(x_{n}\right)-A v_{n}\right\|+\delta_{n}\left\|x_{n}-v_{n}\right\| \longrightarrow 0 \quad \text { as } n \longrightarrow \infty .
\end{aligned}
$$

Using (3.40) and the last inequality, we obtain that

$$
\left\|x_{n}-v_{n}\right\| \leq\left\|x_{n}-x_{n+1}\right\|+\left\|x_{n+1}-v_{n}\right\| \longrightarrow 0 \quad \text { as } n \longrightarrow \infty
$$


From Lemma 2.3(i), (3.59), and (3.61), we have

$$
\begin{aligned}
\left\|T v_{n}-v_{n}\right\| & \leq\left\|T v_{n}-T x_{n}\right\|+\left\|T x_{n}-x_{n}\right\|+\left\|x_{n}-v_{n}\right\| \\
& \leq\left(1+\frac{1+k}{1-k}\right)\left\|v_{n}-x_{n}\right\|+\left\|T x_{n}-x_{n}\right\| \longrightarrow 0 \quad \text { as } n \longrightarrow \infty
\end{aligned}
$$

Hence (3.36) is proved.

Step 5. We claim that

$$
\limsup _{n \rightarrow \infty}\left\langle(A-\gamma f) z, z-v_{n}\right\rangle \leq 0
$$

We choose a subsequence $\left\{v_{n_{i}}\right\}$ of $\left\{v_{n}\right\}$ such that

$$
\lim _{i \rightarrow \infty}\left\langle(A-\gamma f) z, z-v_{n_{i}}\right\rangle=\limsup _{n \rightarrow \infty}\left\langle(A-\gamma f) z, z-v_{n}\right\rangle
$$

Since $\left\{v_{n_{i}}\right\}$ is bounded, there exists a subsequence $\left\{v_{n_{i_{j}}}\right\}$ of $\left\{v_{n_{i}}\right\}$ which converges weakly to $q \in C$.

Next, we show that $q \in \Omega:=F(T) \cap \operatorname{VI}(B, C) \cap \operatorname{MEP}(\Theta, \varphi)$.

(a) We first show $q \in F(T)$. In fact, using Lemma 2.3(ii) and (3.36), we obtain that $q \in F(T)$.

(b) Next, we prove $q \in \operatorname{VI}(B, C)$. For this purpose, let $S$ be the maximal monotone mapping defined by (2.6):

$$
S v= \begin{cases}B v+N_{C} v, & v \in C ; \\ \emptyset, & v \notin C .\end{cases}
$$

For any given $(v, w) \in G(S)$, hence $w-B v \in N_{C}(v)$. Since $v_{n} \in C$, we have

$$
\left\langle v-v_{n}, w-B v\right\rangle \geq 0
$$

On the other hand, from $v_{n}=P_{C}\left(y_{n}-\lambda_{n} B y_{n}\right)$, we have

$$
\left\langle v-v_{n}, v_{n}-\left(y_{n}-\lambda_{n} B y_{n}\right)\right\rangle \geq 0
$$

that is,

$$
\left\langle v-v_{n}, \frac{v_{n}-y_{n}}{\lambda_{n}}+B y_{n}\right\rangle \geq 0
$$


Therefore, we obtian

$$
\begin{aligned}
\left\langle v-v_{n_{i}}, w\right\rangle & \geq\left\langle v-v_{n_{i}}, B v\right\rangle \geq\left\langle v-v_{n_{i}}, B v\right\rangle-\left\langle v-v_{n_{i}}, \frac{v_{n_{i}}-y_{n_{i}}}{\lambda_{n_{i}}}+B y_{n_{i}}\right\rangle \\
& =\left\langle v-v_{n_{i}}, B v-B y_{n_{i}}-\frac{v_{n_{i}}-y_{n_{i}}}{\lambda_{n_{i}}}\right\rangle \\
& =\left\langle v-v_{n_{i}}, B v-B v_{n_{i}}\right\rangle+\left\langle v-v_{n_{i}}, B v_{n_{i}}-B y_{n_{i}}\right\rangle-\left\langle v-v_{n_{i}}, \frac{v_{n_{i}}-y_{n_{i}}}{\lambda_{n_{i}}}\right\rangle \\
& \geq\left\langle v-v_{n_{i}}, B v_{n_{i}}\right\rangle-\left\langle v-v_{n_{i}}, \frac{v_{n_{i}}-y_{n_{i}}}{\lambda_{n_{i}}}+B y_{n_{i}}\right\rangle \\
& =\left\langle v-v_{n_{i}}, B v_{n_{i}}-B y_{n_{i}}\right\rangle-\left\langle v-v_{n_{i}}, \frac{v_{n_{i}}-y_{n_{i}}}{\lambda_{n_{i}}}\right\rangle .
\end{aligned}
$$

Noting that $\left\|v_{n_{i}}-y_{n_{i}}\right\| \rightarrow 0$ as $i \rightarrow \infty$ and $B$ is Lipschitz continuous, hence from (3.69), we obtain

$$
\langle v-q, w\rangle \geq 0
$$

Since $S$ is maximal monotone, we have $q \in S^{-1} 0$, and hence $q \in \operatorname{VI}(B, C)$.

(c) We show $q \in \operatorname{MEP}(\Theta, \varphi)$. In fact, by $u_{n}=T_{r_{n}} x_{n} \in \operatorname{dom} \varphi$, and we have,

$$
\Theta\left(u_{n}, y\right)+\varphi(y)-\varphi\left(u_{n}\right)+\frac{1}{r_{n}}\left\langle y-u_{n}, u_{n}-x_{n}\right\rangle \geq 0, \quad \forall y \in C
$$

From (A2), we also have

$$
\varphi(y)-\varphi\left(u_{n}\right)+\frac{1}{r_{n}}\left\langle y-u_{n}, u_{n}-x_{n}\right\rangle \geq \Theta\left(y, u_{n}\right), \quad \forall y \in C
$$

and hence

$$
\varphi(y)-\varphi\left(u_{n}\right)+\left\langle y-u_{n_{i}}, \frac{u_{n_{i}}-x_{n_{i}}}{r_{n_{i}}}\right\rangle \geq \Theta\left(y, u_{n_{i}}\right), \quad \forall y \in C
$$


From $\left\|u_{n}-x_{n}\right\| \rightarrow 0,\left\|x_{n}-T v_{n}\right\| \rightarrow 0$, and $\left\|T v_{n}-v_{n}\right\| \rightarrow 0$, we get $u_{n_{i}} \rightarrow q$. It follows from (A4), $\left(u_{n_{i}}-x_{n_{i}}\right) / r_{n_{i}} \rightarrow 0$, and the lower semicontinuous of $\varphi$ that

$$
\Theta(y, z)+\varphi(q)-\varphi(y) \leq 0 \quad \forall y \in C
$$

For $t$ with $0<t \leq 1$ and $y \in C$, let $y_{t}=t y+(1-t) q$. Since $y \in C$ and $q \in C$, we have $y_{t} \in C$ and hence $\Theta\left(y_{t}, q\right)+\varphi(q)-\varphi\left(y_{t}\right) \leq 0$. So, from (A1) and (A4) and the convexity of $\varphi$, we have

$$
\begin{aligned}
0 & =\Theta\left(y_{t}, y_{t}\right)+\varphi\left(y_{t}\right)-\varphi\left(y_{t}\right) \\
& \leq t \Theta\left(y_{t}, y\right)+(1-t) \Theta\left(y_{t}, q\right)+t \varphi(y)+(1-t) \varphi(\mathrm{q})-\varphi\left(y_{t}\right) \\
& \leq t\left[\Theta\left(y_{t}, y\right)+\varphi(y)-\varphi\left(y_{t}\right)\right] .
\end{aligned}
$$

Dividing by $t$, we have

$$
\Theta\left(y_{t}, y\right)+\varphi(y)-\varphi\left(y_{t}\right) \geq 0, \quad \forall y \in C
$$

Letting $t \rightarrow 0$, it follows from the weakly semicontinuity of $\varphi$ that

$$
\Theta(q, y)+\varphi(y)-\varphi(q) \geq 0, \quad \forall y \in C
$$

Hence $q \in \operatorname{MEP}(\Theta, \varphi)$. Therefore, the conclusion $q \in \Omega:=F(T) \cap \operatorname{VI}(B, C) \cap \operatorname{MEP}(\Theta, \varphi)$ is proved.

$$
\text { Consequently }
$$

$$
\limsup _{n \rightarrow \infty}\left\langle(A-\gamma f) z, z-v_{n}\right\rangle=\lim _{i \rightarrow \infty}\left\langle(A-\gamma f) z, z-v_{n_{i}}\right\rangle=\langle(A-\gamma f) z, z-q\rangle \leq 0
$$

as required. This together with (3.40) implies that

$$
\begin{aligned}
\limsup _{n \rightarrow \infty}\left\langle\gamma f(z)-A z, x_{n}-z\right\rangle & =\limsup _{n \rightarrow \infty}\left\langle\gamma f(z)-A z,\left(x_{n}-v_{n}\right)+\left(v_{n}-z\right)\right\rangle \\
& \leq \limsup _{n \rightarrow \infty}\left\langle\gamma f(z)-A z, v_{n}-z\right\rangle \\
& \leq 0
\end{aligned}
$$


Fixed Point Theory and Applications

Step 6. Finally, we show that $x_{n} \rightarrow z, y_{n} \rightarrow z, u_{n} \rightarrow z$. Indeed, we note that

$$
\begin{aligned}
\left\|x_{n+1}-z\right\|^{2}= & \left\|\alpha_{n} \gamma f\left(x_{n}\right)+\delta_{n} x_{n}+\left(\left(1-\delta_{n}\right) I-\alpha_{n} A\right) v_{n}-z\right\|^{2} \\
= & \left\|\left(\left(1-\delta_{n}\right) I-\alpha_{n} A\right)\left(v_{n}-z\right)+\delta_{n}\left(x_{n}-z\right)+\alpha_{n}\left(\gamma f\left(x_{n}\right)-A z\right)\right\|^{2} \\
= & \left\|\left(\left(1-\delta_{n}\right) I-\alpha_{n} A\right)\left(v_{n}-z\right)+\delta_{n}\left(x_{n}-z\right)\right\|^{2}+\alpha_{n}^{2}\left\|\gamma f\left(x_{n}\right)-A z\right\|^{2} \\
& +2 \delta_{n} \alpha_{n}\left\langle x_{n}-z, \gamma f\left(x_{n}\right)-A z\right\rangle \\
& +2 \alpha_{n}\left\langle\left(\left(1-\delta_{n}\right) I-\alpha_{n} A\right)\left(v_{n}-z\right), \gamma f\left(x_{n}\right)-A z\right\rangle \\
\leq & \left(\left(1-\delta_{n}-\alpha_{n} \bar{\gamma}\right)\left\|v_{n}-z\right\|+\delta_{n}\left\|x_{n}-z\right\|\right)^{2}+\alpha_{n}^{2}\left\|\gamma f\left(x_{n}\right)-A z\right\|^{2} \\
& +2 \delta_{n} \alpha_{n} \gamma\left\langle x_{n}-z, f\left(x_{n}\right)-f(z)\right\rangle+2 \delta_{n} \alpha_{n}\left\langle x_{n}-z, \gamma f(z)-A(z)\right\rangle \\
& +2\left(1-\delta_{n}\right) \gamma \alpha_{n}\left\langle v_{n}-z, f\left(x_{n}\right)-f(z)\right\rangle+2\left(1-\delta_{n}\right) \alpha_{n}\left\langle v_{n}-z, \gamma f(z)-A z\right\rangle \\
& -2 \alpha_{n}^{2}\left\langle A\left(v_{n}-z\right), \gamma f(z)-A z\right\rangle \\
\leq & \left.\left(\left(1-\delta_{n}-\alpha_{n} \bar{\gamma}\right)\left\|x_{n}-z\right\|+\delta_{n}\left\|x_{n}-z\right\|\right)^{2}+\alpha_{n}^{2} \| \gamma f\left(x_{n}\right)-A z\right) \|^{2} \\
& +2 \delta_{n} \alpha_{n} \gamma \alpha\left\|x_{n}-z\right\|^{2}+2 \delta_{n} \alpha_{n}\left\langle x_{n}-z, \gamma f(q)-A z\right\rangle \\
& +2\left(1-\delta_{n}\right) \gamma \alpha_{n} \alpha\left\|x_{n}-z\right\|^{2}+2\left(1-\delta_{n}\right) \alpha_{n}\left\langle v_{n}-z, \gamma f(z)-A z\right\rangle \\
& -2 \alpha_{n}^{2}\left\langle A\left(v_{n}-z\right), \gamma f(q)-A z\right\rangle \\
= & \left.\left(1-\alpha_{n} \bar{\gamma}\right)^{2}+2 \delta_{n} \alpha_{n} \gamma \alpha+2\left(1-\delta_{n}\right) \gamma \alpha_{n} \alpha\right]\left\|x_{n}-z\right\|^{2}+\alpha_{n}^{2}\left\|\gamma f\left(x_{n}\right)-A z\right\|^{2} \\
& +2 \delta_{n} \alpha_{n}\left\langle x_{n}-z, \gamma f(z)-A z\right\rangle+2\left(1-\delta_{n}\right) \alpha_{n}\left\langle v_{n}-z, \gamma f(z)-A z\right\rangle \\
& -2 \alpha_{n}^{2}\left\langle A\left(v_{n}-z\right), \gamma f(z)-A z\right\rangle \\
\leq & {\left[1-2\left(\bar{\gamma}-\alpha_{n} \gamma\right) \alpha_{n}\right]\left\|x_{n}-z\right\|^{2}+\bar{\gamma}^{2} \alpha_{n}^{2}\left\|x_{n}-z\right\|^{2}+\alpha_{n}^{2}\left\|\gamma f\left(x_{n}\right)-A z\right\|^{2} } \\
& +2 \delta_{n} \alpha_{n}\left\langle x_{n}-z, \gamma f(z)-A z\right\rangle+2\left(1-\delta_{n}\right) \alpha_{n}\left\langle v_{n}-z, \gamma f(z)-A z\right\rangle \\
& +2 \alpha_{n}^{2}\left\|A\left(v_{n}-z\right)\right\|\|\gamma f(z)-A z\| \\
= & {\left[1-2\left(\bar{\gamma}-\alpha_{n} \gamma\right) \alpha_{n}\right]\left\|x_{n}-z\right\|^{2} } \\
& +\alpha_{n}\left\{\alpha _ { n } \left(\bar{\gamma}^{2}\left\|x_{n}-z\right\|^{2}+\left\|\gamma f\left(x_{n}\right)-A z\right\|^{2}\right.\right. \\
& \left.+2\left\|A\left(v_{n}-z\right)\right\|\|\gamma f(z)-A z\|\right)+2 \delta_{n}\left\langle x_{n}-z, \gamma f(z)-A z\right\rangle \\
& +A z\rangle\} . \\
& \\
& \\
&
\end{aligned}
$$

Since $\left\{x_{n}\right\},\left\{f\left(x_{n}\right)\right\}$, and $\left\{v_{n}\right\}$ are bounded, we can take a constant $K>0$ such that

$$
\bar{\gamma}^{2}\left\|x_{n}-z\right\|^{2}+\left\|\gamma f\left(x_{n}\right)-A z\right\|^{2}+2\left\|A\left(v_{n}-z\right)\right\|\|\gamma f(z)-A z\| \leq K
$$


for all $n \geq 0$. It then follows that

$$
\left\|x_{n+1}-z\right\|^{2} \leq\left[1-2\left(\bar{\gamma}-\alpha_{n} \gamma\right) \alpha_{n}\right]\left\|x_{n}-z\right\|^{2}+\alpha_{n} \sigma_{n}
$$

where

$$
\sigma_{n}=2 \delta_{n}\left\langle x_{n}-z, \gamma f(z)-A z\right\rangle+2\left(1-\delta_{n}\right)\left\langle v_{n}-z, \gamma f(z)-A z\right\rangle+\alpha_{n} K
$$

Using (D1), and (3.79), we get $\lim _{\sup _{n \rightarrow \infty}} \delta_{n} \leq 0$. Now applying Lemma 2.1 to (3.82), we conclude that $x_{n} \rightarrow z$. From $\left\|x_{n}-y_{n}\right\| \rightarrow 0$ and $\left\|x_{n}-u_{n}\right\| \rightarrow 0$, we obtain $y_{n} \rightarrow z, u_{n} \rightarrow z$. The proof is now complete.

By Theorem 3.1, we can obtain some new and interesting strong convergence theorems. Now we give some examples as follows.

Setting $\varphi=0$ in Theorem 3.1, we have the following result.

Corollary 3.2. Let $C$ be a nonempty closed convex subset of a Hilbert space $H$. Let $\Theta$ be a bifunction from $C \times C$ to $\mathbb{R}$ satifies (A1)-(A4). Let $T$ be a $k$-strictly pseudocontractive mapping of $C$ into itself. Let $f$ be a contraction of $C$ into itself with coefficient $\beta \in(0,1), B$ an $\alpha$-inverse-strongly monotone mapping of $C$ into $H$ such that $\Omega:=F(T) \cap V I(B, C) \cap E P(\Theta) \neq \emptyset$. Let $A$ be a strongly bounded linear self-adjoint operator with coefficient $\bar{\gamma}>0$ and $0<\gamma<\bar{\gamma} / \beta$. Given the sequences $\left\{\alpha_{n}\right\},\left\{\beta_{n}\right\},\left\{\delta_{n}\right\},\left\{\lambda_{n}\right\}$, and $\left\{r_{n}\right\}$ in $[0,1]$ satisfies the following conditions

(D1) $\lim _{n \rightarrow \infty} \alpha_{n}=0, \sum_{n=1}^{\infty} \alpha_{n}=\infty$;

(D2) $0<\liminf _{n \rightarrow \infty} \delta_{n} \leq \limsup _{n \rightarrow \infty} \delta_{n}<1$;

(D3) $0 \leq k \leq \beta_{n}<\varepsilon<1$ for all $n \geq 0$, and $\lim _{n \rightarrow \infty}\left|\beta_{n+1}-\beta_{n}\right|=0$;

(D4) $\left\{\lambda_{n}\right\} \subset[a, b]$ for some $a, b$ with $0<a<b<2 \alpha$, and $\lim _{n \rightarrow \infty}\left|\lambda_{n+1}-\lambda_{n}\right|=0$

(D5) $\liminf _{n \rightarrow \infty} r_{n}>0, \lim _{n \rightarrow \infty}\left|r_{n+1}-r_{n}\right|=0$.

Let $\left\{x_{n}\right\},\left\{u_{n}\right\}$, and $\left\{y_{n}\right\}$ be sequences generated by

$$
\begin{gathered}
x_{1}=x \in C \text { chosen arbitrarily, } \\
\Theta\left(u_{n}, y\right)+\frac{1}{r_{n}}\left\langle y-u_{n}, u_{n}-x_{n}\right\rangle \geq 0, \quad \forall y \in C, \\
y_{n}=\beta_{n} u_{n}+\left(1-\beta_{n}\right) T u_{n}, \\
x_{n+1}=\alpha_{n} \gamma f\left(x_{n}\right)+\delta_{n} x_{n}+\left(\left(1-\delta_{n}\right) I-\alpha_{n} A\right) P_{C}\left(y_{n}-\lambda_{n} B y_{n}\right), \quad n \geq 1 .
\end{gathered}
$$

Then $\left\{x_{n}\right\},\left\{u_{n}\right\}$ and $\left\{y_{n}\right\}$ converge strongly to a point $z \in \Omega$ which is the unique solution of the variational inequality

$$
\langle(A-r f) z, z-x\rangle \leq 0, \quad \forall x \in \Omega
$$

Equivalently, one has $z=P_{\Omega}(I-A+\gamma f)(z)$. 
Setting $\Theta=0, r_{n}=1$ and $\varphi=0$ in Theorem 3.1, we have $x_{n}=u_{n}$, then the following result is obtained.

Corollary 3.3. Let $C$ be a nonempty closed convex subset of a Hilbert space $H$. Let $T$ be a $k$-strictly pseudocontractive mapping of $C$ into itself. Let $f$ be a contraction of $C$ into itself with coefficient $\beta \in$ $(0,1), B$ an $\alpha$-inverse-strongly monotone mapping of $C$ into $H$ such that $\Omega:=F(T) \cap V I(B, C) \neq \emptyset$. Let $A$ be a strongly bounded linear self-adjoint operator with coefficient $\bar{\gamma}>0$ and $0<\gamma<\bar{\gamma} / \beta$. Given the sequences $\left\{\alpha_{n}\right\},\left\{\beta_{n}\right\},\left\{\delta_{n}\right\}$ and $\left\{\lambda_{n}\right\}$ in $[0,1]$ satifies the following conditions

(D1) $\lim _{n \rightarrow \infty} \alpha_{n}=0, \sum_{n=1}^{\infty} \alpha_{n}=\infty$;

(D2) $0<\liminf _{n \rightarrow \infty} \delta_{n} \leq \lim \sup _{n \rightarrow \infty} \delta_{n}<1$;

(D3) $0 \leq k \leq \beta_{n}<\varepsilon<1$ for all $n \geq 0$, and $\lim _{n \rightarrow \infty}\left|\beta_{n+1}-\beta_{n}\right|=0$;

(D4) $\left\{\lambda_{n}\right\} \subset[a, b]$ for some $a, b$ with $0<a<b<2 \alpha$ and $\lim _{n \rightarrow \infty}\left|\lambda_{n+1}-\lambda_{n}\right|=0$.

Let $\left\{x_{n}\right\}$ and $\left\{y_{n}\right\}$ be sequences generated by

$$
\begin{gathered}
x_{1}=x \in C \text { chosen arbitrarily, } \\
y_{n}=\beta_{n} x_{n}+\left(1-\beta_{n}\right) T x_{n}, \\
x_{n+1}=\alpha_{n} \gamma f\left(x_{n}\right)+\delta_{n} x_{n}+\left(\left(1-\delta_{n}\right) I-\alpha_{n} A\right) P_{C}\left(y_{n}-\lambda_{n} B y_{n}\right), \quad n \geq 1 .
\end{gathered}
$$

Then $\left\{x_{n}\right\}$ and $\left\{y_{n}\right\}$ converge strongly to a point $z \in \Omega$ which is the unique solution of the variational inequality

$$
\langle(A-\gamma f) z, z-x\rangle \leq 0, \quad \forall x \in \Omega .
$$

Equivalently, one has $z=P_{\Omega}(I-A+\gamma f)(z)$.

Remark 3.4. (i) Since the conditions (C1) and (C2) have been weakened by the conditions (D1) and (D3) respectively. Theorem 3.1 and Corollary 3.2 generalize and improve [44, Theorem 3.2].

(ii) We can remove the control condition $\lim _{n \rightarrow \infty} \beta_{n}=\varepsilon$ on the parameter $\left\{\beta_{n}\right\}$ in $\left(C^{\prime} 2\right)$.

(iii) Since the conditions (C1) and (C2) have been weakened by the conditions (D1) and (D3) respectively. Theorem 3.1 and Corollary 3.3 generalize and improve [43, Theorem 2.1].

Setting $\varphi=0, \beta_{n}=0, B=0$ and $T$ is nonexpansive in Theorem 3.1, we have the following result.

Corollary 3.5. Let $C$ be a nonempty closed convex subset of a Hilbert space $H$. Let $\Theta$ be a bifunction from $C \times C$ to $\mathbb{R}$ satifies (A1)-(A4). Let $T$ be a nonexpansive mapping of $C$ into itself. Let $f$ be a contraction of $C$ into itself with coefficient $\beta \in(0,1)$ such that $\Omega:=F(T) \cap E P(\Theta) \neq \emptyset$. Let $A$ be a strongly bounded linear self-adjoint operator with coefficient $\bar{\gamma}>0$ and $0<\gamma<\bar{\gamma} / \beta$. Given the sequences $\left\{\alpha_{n}\right\},\left\{\delta_{n}\right\}$, and $\left\{r_{n}\right\}$ in $[0,1]$ satifies the following conditions

(D1) $\lim _{n \rightarrow \infty} \alpha_{n}=0, \sum_{n=1}^{\infty} \alpha_{n}=\infty$;

(D2) $0<\liminf _{n \rightarrow \infty} \delta_{n} \leq \lim \sup _{n \rightarrow \infty} \delta_{n}<1$;

(D3) $\liminf _{n \rightarrow \infty} r_{n}>0, \lim _{n \rightarrow \infty}\left|r_{n+1}-r_{n}\right|=0$. 
Let $\left\{x_{n}\right\},\left\{u_{n}\right\}$, and $\left\{y_{n}\right\}$ be sequences generated by

$$
\begin{gathered}
x_{1}=x \in C \text { chosen arbitrarily, } \\
\Theta\left(u_{n}, y\right)+\frac{1}{r_{n}}\left\langle y-u_{n}, u_{n}-x_{n}\right\rangle \geq 0, \quad \forall y \in C, \\
x_{n+1}=\alpha_{n} \gamma f\left(x_{n}\right)+\delta_{n} x_{n}+\left(\left(1-\delta_{n}\right) I-\alpha_{n} A\right) T u_{n}, \quad n \geq 1 .
\end{gathered}
$$

Then $\left\{x_{n}\right\},\left\{u_{n}\right\}$ and $\left\{y_{n}\right\}$ converge strongly to a point $z \in \Omega$ which is the unique solution of the variational inequality

$$
\langle(A-\gamma f) z, z-x\rangle \leq 0, \quad \forall x \in \Omega .
$$

Equivalently, one has $z=P_{\Omega}(I-A+\gamma f)(z)$.

Remark 3.6. Since the conditions $\sum_{n=1}^{\infty}\left|\alpha_{n+1}-\alpha_{n}\right|<\infty$ and $\sum_{n=1}^{\infty}\left|r_{n+1}-r_{n}\right|<\infty$ have been weakened by the conditions $\lim _{n \rightarrow \infty}\left|\alpha_{n+1}-\alpha_{n}\right|=0$ and $\lim _{n \rightarrow \infty}\left|r_{n+1}-r_{n}\right|=0$, respectively. Hence Corollary 3.5 generalize, extend and improve [17, Theorem 3.3].

\section{Applications}

First, we will utilize the results presented in this paper to study the following optimization problem:

$$
\min _{y \in C} \varphi(y)
$$

where $C$ is a nonempty bounded closed convex subset of a Hilbert space and $\varphi: C \rightarrow \mathbb{R} \cup$ $\{+\infty\}$ is a proper convex and lower semicontinuous function. We denote by $\operatorname{Argmin}(\varphi)$ the set of solutions in (4.1). Let $\Theta(x, y)=0$ for all $x, y \in C, \gamma \equiv 1, A \equiv I, T=I$ and $f:=x$ in Theorem 3.1, then $\operatorname{MEP}(\Theta, \varphi)=\operatorname{Argmin}(\varphi)$. It follows from Theorem 3.1 that the iterative sequence $\left\{x_{n}\right\}$ is defined by

$$
\begin{gathered}
x_{1}=x \in C \text { chosen arbitrarily, } \\
u_{n}=\underset{y \in C}{\operatorname{argmin}}\left\{\varphi(y)+\frac{1}{2 r_{n}}\left\|y-x_{n}\right\|^{2}\right\}, \\
x_{n+1}=\alpha_{n} x+\delta_{n} x_{n}+\left(1-\delta_{n}-\alpha_{n}\right) P_{C}\left(u_{n}-\lambda_{n} B u_{n}\right), \quad n \geq 1,
\end{gathered}
$$

where $\left\{\alpha_{n}\right\},\left\{\delta_{n}\right\} \subseteq[0,1],\left\{\lambda_{n}\right\},\left\{r_{n}\right\} \subseteq(0,1)$ satisfy the conditions (D1)-(D5) in Theorem 3.1. Then the sequence $\left\{x_{n}\right\}$ converges strongly to a solution $z=P_{\mathrm{VI}(A, C) \cap \operatorname{Argmin}(\varphi)} x$. 
Let $\Theta(x, y)=0$ for all $x, y \in C, T=I, \gamma \equiv 1, A \equiv I, f:=x$ and $B \equiv 0$ in Theorem 3.1, then $\operatorname{MEP}(\Theta, \varphi)=\operatorname{Argmin}(\varphi)$. It follows from Theorem 3.1 that the iterative sequence $\left\{x_{n}\right\}$ defined by

$$
\begin{gathered}
x_{1}=x \in C \text { chosen arbitrarily, } \\
u_{n}=\underset{y \in C}{\operatorname{argmin}}\left\{\varphi(y)+\frac{1}{2 r_{n}}\left\|y-x_{n}\right\|^{2}\right\}, \\
x_{n+1}=\alpha_{n} x+\delta_{n} x_{n}+\left(1-\delta_{n}-\alpha_{n}\right) u_{n}, \quad \forall n \geq 1,
\end{gathered}
$$

where $\left\{\alpha_{n}\right\},\left\{\delta_{n}\right\} \subseteq[0,1]$, and $\left\{r_{n}\right\} \subseteq(0, \infty)$ satisfy the conditions (D1), (D2) and (D5), respectively in Theorem 3.1. Then the sequence $\left\{x_{n}\right\}$ converges strongly to a solution $z=$ $P_{\operatorname{Argmin}(\varphi)} x$.

We remark that the algorithms (4.2) and (4.3) are variants of the proximal method for optimization problems introduced and studied by Martinet [49], Rockafellar [45], Ferris [50] and many others.

Next, we give the strong convergence theorem for finding a common element of the set of common fixed point of a finite family of strictly pseudocontractive mappings, the set of solutions of the variational inequality problem and the set of solutions of the mixed equilibrium problem in a Hilbert space.

Theorem 4.1. Let $C$ be a nonempty closed convex subset of a Hilbert space $H$. Let $\Theta$ be a bifunction from $C \times C$ to $\mathbb{R}$ satifies $(A 1)-(A 4)$ and $\varphi: C \rightarrow \mathbb{R} \cup\{+\infty\}$ be a proper lower semicontinuous and convex function. For each $i=1,2, \ldots, N$, let $T_{i}$ be a $k_{i}$-strictly pseudocontractive mapping of $C$ into itself for some $0 \leq k_{i}<1$. Let $f$ be a contraction of $C$ into itself with coefficient $\beta \in(0,1)$, $B$ an $\alpha$-inverse-strongly monotone mapping of $C$ into $H$ such that $\Omega:=\bigcap_{i=1}^{N} F\left(T_{i}\right) \cap V I(B, C) \cap$ $\operatorname{MEP}(\Theta, \varphi) \neq \emptyset$. Let $A$ be a strongly bounded linear self-adjoint operator with coefficient $\bar{\gamma}>0$ and $0<\gamma<\bar{\gamma} / \beta$. Assume that either (B1) or (B2) holds. Given the sequences $\left\{\alpha_{n}\right\},\left\{\beta_{n}\right\},\left\{\delta_{n}\right\},\left\{\lambda_{n}\right\}$ and $\left\{r_{n}\right\}$ in $[0,1]$ satifies the following conditions

(D1) $\lim _{n \rightarrow \infty} \alpha_{n}=0, \sum_{n=1}^{\infty} \alpha_{n}=\infty$;

(D2) $0<\liminf _{n \rightarrow \infty} \delta_{n} \leq \limsup _{n \rightarrow \infty} \delta_{n}<1$;

(D3) $0 \leq \max \left\{k_{i}: i=1,2, \ldots, N\right\} \leq \beta_{n}<\beta<1$ for all $n \geq 0$, and $\lim _{n \rightarrow \infty}\left|\beta_{n+1}-\beta_{n}\right|=0$;

(D4) $\left\{\lambda_{n}\right\} \subset[a, b]$ for some $a, b$ with $0<a<b<2 \alpha$ and $\lim _{n \rightarrow \infty}\left|\lambda_{n+1}-\lambda_{n}\right|=0$;

(D5) $\liminf _{n \rightarrow \infty} r_{n}>0, \lim _{n \rightarrow \infty}\left|r_{n+1}-r_{n}\right|=0$.

Let $\left\{x_{n}\right\},\left\{u_{n}\right\}$ and $\left\{y_{n}\right\}$ be sequences generated by

$$
\begin{gathered}
x_{0}=x \in C \text { chosen arbitrarily, } \\
\Theta\left(u_{n}, y\right)+\varphi(y)-\varphi\left(u_{n}\right)+\frac{1}{r_{n}}\left\langle y-u_{n}, u_{n}-x_{n}\right\rangle \geq 0, \quad \forall y \in C, \\
y_{n}=\beta_{n} u_{n}+\left(1-\beta_{n}\right) \sum_{i=1}^{N} \eta_{i} T_{i} u_{n} \\
x_{n+1}=\alpha_{n} \gamma f\left(x_{n}\right)+\delta_{n} x_{n}+\left(\left(1-\delta_{n}\right) I-\alpha_{n} A\right) P_{C}\left(y_{n}-\lambda_{n} B y_{n}\right), \quad n \geq 1,
\end{gathered}
$$


where $\eta_{i}$ is a positive constant such that $\eta_{1}+\eta_{2}+\cdots+\eta_{N}=1$. Then both $\left\{x_{n}\right\},\left\{u_{n}\right\}$ and $\left\{y_{n}\right\}$ converge strongly to a point $z \in \Omega$ which is the unique solution of the variational inequality

$$
\langle(A-\gamma f) z, z-x\rangle \leq 0, \quad x \in \Omega \text {. }
$$

Equivalently, one has $z=P_{\Omega}(I-A+\gamma f)(z)$.

Proof. Let $\left\{\eta_{i}\right\}_{i=1}^{N} \subset(0,1)$ such that $\sum_{i=1}^{N} \eta_{i}=1$ and define $T x=\sum_{i=1}^{N} \eta_{i} T_{i} x$. By Lemmas 2.5 and 2.6, we conclude that $T: C \rightarrow C$ is a $k$-strictly pseudocontractive mapping with $k=\max \left\{k_{i}\right.$ : $1 \leq i \leq N\}$ and $F(T)=F\left(\sum_{i=1}^{N} \eta_{i} T_{i}\right)=\bigcap_{i=1}^{N} F\left(T_{i}\right)$. From Theorem 3.1, we can obtain the desired conclusion easily.

Finally, we will apply the main results to the problem for finding a common element of the set of fixed points of two finite families of $k$-strictly pseudocontractive mappings, the set of solutions of the variational inequality and the set of solutions of the mixed equilibrium problem.

Let $S_{i}: C \rightarrow H$ be a $k_{i}$-strictly pseudocontractive mapping for some $0 \leq k_{i}<1$. We define a mapping $B=I-\sum_{i=1}^{N} \xi_{i} S_{i}: C \rightarrow H$ where $\left\{\xi_{i}\right\}_{i=1}^{N}$ is a positive sequence such that $\sum_{i=1}^{N} \xi_{i}=1$, then $B$ is a $(1-k) / 2$-inverse-strongly monotone mapping with $k=\max \left\{k_{i}: 1 \leq\right.$ $i \leq N\}$. In fact, from Lemma 2.5, we have

$$
\left\|\sum_{i=1}^{N} \xi_{i} S_{i} x-\sum_{i=1}^{N} \xi_{i} S_{i} y\right\|^{2} \leq\|x-y\|^{2}+k\left\|\left(I-\sum_{i=1}^{N} \xi_{i} S_{i}\right) x-\left(I-\sum_{i=1}^{N} \xi_{i} S_{i}\right) y\right\|^{2}, \quad \forall x, y \in C .
$$

That is

$$
\|(I-B) x-(I-B) y\|^{2} \leq\|x-y\|^{2}+k\|B x-B y\|^{2} .
$$

On the other hand

$$
\|(I-B) x-(I-B) y\|^{2}=\|x-y\|^{2}-2\langle x-y, B x-B y\rangle+\|B x-B y\|^{2} .
$$

Hence we have

$$
\langle x-y, B x-B y\rangle \geq \frac{1-k}{2}\|B x-B y\|^{2}
$$

This shows that $B$ is $(1-k) / 2$-inverse-strongly monotone.

Theorem 4.2. Let $C$ be a nonempty closed convex subset of a Hilbert space $H$. Let $\Theta$ be a bifunction from $C \times C$ to $\mathbb{R}$ satifies $(A 1)-(A 4)$ and $\varphi: C \rightarrow \mathbb{R} \cup\{+\infty\}$ be a proper lower semicontinuous and convex function. Let $\left\{T_{1}, T_{2}, \ldots, T_{N}\right\}$ be a finite family of $k_{i}^{T}$-strictly pseudocontractive mapping of $C$ into itself and $\left\{S_{1}, S_{2}, \ldots, S_{N}\right\}$ be a finite family of $k_{i}^{S}$-strictly pseudocontractive mapping of $C$ into $H$ for some $k_{i}^{T}, k_{i}^{S} \in(0,1)$ such that $\Omega:=\bigcap_{i=1}^{N} F\left(T_{i}\right) \cap \bigcap_{i=1}^{N} F\left(S_{i}\right) \cap \operatorname{MEP}(\Theta, \varphi) \neq \emptyset$. Let $f$ be a contraction of $C$ into itself with coefficient $\beta \in(0,1)$. Let $A$ be a strongly bounded linear self-adjoint 
operator with coefficient $\bar{\gamma}>0$ and $0<\gamma<\bar{\gamma} / \beta$. Assume that either (B1) or (B2) holds. Given the sequences $\left\{\alpha_{n}\right\},\left\{\beta_{n}\right\},\left\{\delta_{n}\right\},\left\{\lambda_{n}\right\}$ and $\left\{r_{n}\right\}$ in $[0,1]$ satifies the following conditions

(D1) $\lim _{n \rightarrow \infty} \alpha_{n}=0, \sum_{n=1}^{\infty} \alpha_{n}=\infty$;

(D2) $0<\liminf _{n \rightarrow \infty} \delta_{n} \leq \limsup _{n \rightarrow \infty} \delta_{n}<1$;

(D3) $0 \leq \max _{1 \leq i \leq N} k_{i}^{T} \leq \beta_{n}<\beta<1$ and $0 \leq \max _{1 \leq i \leq N} k_{i}^{S} \leq \beta_{n}<\beta<1$ for all $n \geq 0$, and $\lim _{n \rightarrow \infty}\left|\beta_{n+1}-\beta_{n}\right|=0$;

(D4) $\left\{\lambda_{n}\right\} \subset[a, b]$ for some $a, b$ with $0<a<b<2 \alpha$ and $\lim _{n \rightarrow \infty}\left|\lambda_{n+1}-\lambda_{n}\right|=0$;

(D5) $\liminf _{n \rightarrow \infty} r_{n}>0, \lim _{n \rightarrow \infty}\left|r_{n+1}-r_{n}\right|=0$.

Let $\left\{x_{n}\right\},\left\{u_{n}\right\}$ and $\left\{y_{n}\right\}$ be sequences generated by

$$
\begin{gathered}
x_{1}=x \in C \text { chosen arbitrarily, } \\
\Theta\left(u_{n}, y\right)+\varphi(y)-\varphi\left(u_{n}\right)+\frac{1}{r_{n}}\left\langle y-u_{n}, u_{n}-x_{n}\right\rangle \geq 0, \quad \forall y \in C, \\
y_{n}=\beta_{n} u_{n}+\left(1-\beta_{n}\right) \sum_{i=1}^{N} \eta_{i} T_{i} u_{n} \\
x_{n+1}=\alpha_{n} \gamma f\left(x_{n}\right)+\delta_{n} x_{n}+\left(\left(1-\delta_{n}\right) I-\alpha_{n} A\right) P_{C}\left(\left(1-\lambda_{n}\right) y_{n}-\lambda_{n} \sum_{i=1}^{N} \xi_{i} S_{i} y_{n}\right), \quad n \geq 1,
\end{gathered}
$$

where $\eta_{i}$ and $\xi_{i}$ are positive constants such that $\sum_{i=1}^{N} \eta_{i}=1$ and $\sum_{i=1}^{N} \xi_{i}=1$, respectively. Then $\left\{x_{n}\right\},\left\{u_{n}\right\}$ and $\left\{y_{n}\right\}$ converge strongly to a point $z \in \Omega$ which is the unique solution of the variational inequality

$$
\langle(A-\gamma f) z, z-x\rangle \leq 0, \quad x \in \Omega
$$

Equivalently, we have $z=P_{\Omega}(I-A+\gamma f)(z)$.

Proof. Taking $B=I-\sum_{i=1}^{N} \xi_{i} S_{i}: C \rightarrow H$ in Theorem 4.1, we know that $B: C \rightarrow H$ is $\alpha$ inverse strongly monotone with $\alpha=(1-k) / 2$. Hence, $B$ is a monotone $L$-Lipschitz continuous mapping with $L=2 /\left(1-k^{T}\right)$. From Lemma 2.6, we know that $\sum_{i=1}^{N} \xi_{i} S_{i}$ is a $k^{T}$-strictly pseudocontractive mapping with $k^{T}=\max \left\{k_{i}^{T}: 1 \leq i \leq N\right\}$ and then $F\left(\sum_{i=1}^{N} \xi_{i} S_{i}\right)=\operatorname{VI}(B, C)$ by Lemma 2.6. Observe that

$$
P_{C}\left(y_{n}-\lambda_{n} B y_{n}\right)=P_{C}\left(\left(1-\lambda_{n}\right) y_{n}-\lambda_{n} \sum_{i=1}^{N} \xi_{i} S_{i} y_{n}\right)
$$

The conclusion can be obtained from Theorem 4.1.

\section{Acknowledgments}

R. Wangkeeree would like to thank The National Research Council of Thailand, Grant SCAR-012/2552 for financial support. The authors would like to thank the referees for reading this paper carefully, providing valuable suggestions and comments, and pointing out a major error in the original version of this paper. 


\section{References}

[1] L.-C. Ceng and J.-C. Yao, "A hybrid iterative scheme for mixed equilibrium problems and fixed point problems," Journal of Computational and Applied Mathematics, vol. 214, no. 1, pp. 186-201, 2008.

[2] E. Blum and W. Oettli, "From optimization and variational inequalities to equilibrium problems," The Mathematics Student, vol. 63, no. 1-4, pp. 123-145, 1994.

[3] H. Iiduka and W. Takahashi, "Strong convergence theorems for nonexpansive mappings and inversestrongly monotone mappings," Nonlinear Analysis: Theory, Methods E Applications, vol. 61, no. 3, pp. 341-350, 2005.

[4] P. Kumam, "A new hybrid iterative method for solution of equilibrium problems and fixed point problems for an inverse strongly monotone operator and a nonexpansive mapping," Journal of Applied Mathematics and Computing, vol. 29, no. 1-2, pp. 263-280, 2009.

[5] W. Kumam and P. Kumam, "Hybrid iterative scheme by a relaxed extragradient method for solutions of equilibrium problems and a general system of variational inequalities with application to optimization," Nonlinear Analysis: Hybrid Systems, vol. 3, no. 4, pp. 640-656, 2009.

[6] W. Takahashi and M. Toyoda, "Weak convergence theorems for nonexpansive mappings and monotone mappings," Journal of Optimization Theory and Applications, vol. 118, no. 2, pp. 417-428, 2003.

[7] U. Kamraksa and R. Wangkeeree, "A general iterative method for variational inequality problems and fixed point problems of an infinite family of nonexpansive mappings in Hilbert spaces," Thai Journal of Mathematics, vol. 6, no. 1, pp. 147-170, 2008.

[8] R. Wangkeeree and U. Kamraksa, "A general iterative method for solving the variational inequality problem and fixed point problem of an infinite family of nonexpansive mappings in Hilbert spaces," Fixed Point Theory and Applications, vol. 2009, Article ID 369215, 23 pages, 2009.

[9] R. Wangkeeree, "An extragradient approximation method for equilibrium problems and fixed point problems of a countable family of nonexpansive mappings," Fixed Point Theory and Applications, vol. 2008, Article ID 134148, 17 pages, 2008.

[10] R. Wangkeeree and U. Kamraksa, "An iterative approximation method for solving a general system of variational inequality problems and mixed equilibrium problems," Nonlinear Analysis: Hybrid Systems. In press.

[11] Y. Yao, Y.-C. Liou, and J.-C. Yao, "An extragradient method for fixed point problems and variational inequality problems," Journal of Inequalities and Applications, vol. 2007, Article ID 38752, 12 pages, 2007.

[12] J.-C. Yao and O. Chadli, "Pseudomonotone complementarity problems and variational inequalities," in Handbook of Generalized Convexity and Generalized Monotonicity, J. P. Crouzeix, N. Haddjissas, and S. Schaible, Eds., vol. 76 of Nonconvex Optimization and Its Applications, pp. 501-558, Springer, New York, NY, USA, 2005.

[13] L. C. Zeng, S. Schaible, and J. C. Yao, "Iterative algorithm for generalized set-valued strongly nonlinear mixed variational-like inequalities," Journal of Optimization Theory and Applications, vol. 124, no. 3, pp. 725-738, 2005.

[14] P.L. Combettes and S.A. Hirstoaga, "Equilibrium programming using proximal-like algorithms," Mathematical Programming, vol. 78, no. 1, pp. 29-41, 1997.

[15] S. D. Flåm and A. S. Antipin, "Equilibrium programming using proximal-like algorithms," Mathematical Programming, vol. 78, no. 1, pp. 29-41, 1997.

[16] S. Takahashi and W. Takahashi, "Viscosity approximation methods for equilibrium problems and fixed point problems in Hilbert spaces," Journal of Mathematical Analysis and Applications, vol. 331, no. 1, pp. 506-515, 2007.

[17] S. Plubtieng and R. Punpaeng, "A general iterative method for equilibrium problems and fixed point problems in Hilbert spaces," Journal of Mathematical Analysis and Applications, vol. 336, no. 1, pp. 455469, 2007.

[18] J.-W. Peng and J.-C. Yao, "Strong convergence theorems of iterative scheme based on the extragradient method for mixed equilibrium problems and fixed point problems," Mathematical and Computer Modelling, vol. 49, no. 9-10, pp. 1816-1828, 2009.

[19] F. E. Browder and W. V. Petryshyn, "Construction of fixed points of nonlinear mappings in Hilbert space," Journal of Mathematical Analysis and Applications, vol. 20, pp. 197-228, 1967.

[20] F. Liu and M. Z. Nashed, "Regularization of nonlinear Ill-posed variational inequalities and convergence rates," Set-Valued Analysis, vol. 6, no. 4, pp. 313-344, 1998. 
[21] F. Deutsch and I. Yamada, "Minimizing certain convex functions over the intersection of the fixed point sets of nonexpansive mappings," Numerical Functional Analysis and Optimization, vol. 19, no. 1-2, pp. 33-56, 1998.

[22] H.-K. Xu, "Iterative algorithms for nonlinear operators," Journal of the London Mathematical Society, vol. 66, no. 1, pp. 240-256, 2002.

[23] H.-K. Xu, "An iterative approach to quadratic optimization," Journal of Optimization Theory and Applications, vol. 116, no. 3, pp. 659-678, 2003.

[24] I. Yamada, "The hybrid steepest descent method for the variational inequality problem of the intersection of fixed point sets of nonexpansive mappings," in Inherently Parallel Algorithm for Feasibility and Optimization, D. Butnariu, Y. Censor, and S. Reich, Eds., pp. 473-504, Elsevier, London, UK, 2001.

[25] G. Marino and H.-K. Xu, "A general iterative method for nonexpansive mappings in Hilbert spaces," Journal of Mathematical Analysis and Applications, vol. 318, no. 1, pp. 43-52, 2006.

[26] A. Moudafi, "Viscosity approximation methods for fixed-points problems," Journal of Mathematical Analysis and Applications, vol. 241, no. 1, pp. 46-55, 2000.

[27] W. R. Mann, "Mean value methods in iteration," Proceedings of the American Mathematical Society, vol. 4, pp. 506-510, 1953.

[28] C. Byrne, "A unified treatment of some iterative algorithms in signal processing and image reconstruction," Inverse Problems, vol. 20, no. 1, pp. 103-120, 2004.

[29] K.-K. Tan and H. K. Xu, "Approximating fixed points of nonexpansive mappings by the Ishikawa iteration process," Journal of Mathematical Analysis and Applications, vol. 178, no. 2, pp. 301-308, 1993.

[30] R. Wittmann, "Approximation of fixed points of nonexpansive mappings," Archiv der Mathematik, vol. 58, no. 5, pp. 486-491, 1992.

[31] H.-K. Xu, "Iterative algorithms for nonlinear operators," Journal of the London Mathematical Society, vol. 66, no. 1, pp. 240-256, 2002.

[32] L.-C. Zeng, "A note on approximating fixed points of nonexpansive mappings by the Ishikawa iteration process," Journal of Mathematical Analysis and Applications, vol. 226, no. 1, pp. 245-250, 1998.

[33] S. Reich, "Weak convergence theorems for nonexpansive mappings in Banach spaces," Journal of Mathematical Analysis and Applications, vol. 67, no. 2, pp. 274-276, 1979.

[34] A. Genel and J. Lindenstrauss, "An example concerning fixed points," Israel Journal of Mathematics, vol. 22, no. 1, pp. 81-86, 1975.

[35] G. L. Acedo and H.-K. Xu, "Iterative methods for strict pseudo-contractions in Hilbert spaces," Nonlinear Analysis: Theory, Methods \& Applications, vol. 67, no. 7, pp. 2258-2271, 2007.

[36] T.-H. Kim and H.-K. Xu, "Strong convergence of modified Mann iterations," Nonlinear Analysis: Theory, Methods \& Applications, vol. 61, no. 1-2, pp. 51-60, 2005.

[37] C. Martinez-Yanes and H.-K. Xu, "Strong convergence of the CQ method for fixed point iteration processes," Nonlinear Analysis: Theory, Methods \& Applications, vol. 64, no. 11, pp. 2400-2411, 2006.

[38] K. Nakajo and W. Takahashi, "Strong convergence theorems for nonexpansive mappings and nonexpansive semigroups," Journal of Mathematical Analysis and Applications, vol. 279, no. 2, pp. 372 $379,2003$.

[39] X. Qin and Y. Su, "Approximation of a zero point of accretive operator in Banach spaces," Journal of Mathematical Analysis and Applications, vol. 329, no. 1, pp. 415-424, 2007.

[40] H. Zhou, "Convergence theorems of fixed points for $\mathcal{\kappa}$-strict pseudo-contractions in Hilbert spaces," Nonlinear Analysis: Theory, Methods \& Applications, vol. 69, no. 2, pp. 456-462, 2008.

[41] Y. Yao, R. Chen, and J.-C. Yao, "Strong convergence and certain control conditions for modified Mann iteration," Nonlinear Analysis: Theory, Methods E Applications, vol. 68, no. 6, pp. 1687-1693, 2008.

[42] G. Marino and H.-K. Xu, "Weak and strong convergence theorems for strict pseudo-contractions in Hilbert spaces," Journal of Mathematical Analysis and Applications, vol. 329, no. 1, pp. 336-346, 2007.

[43] G. Marino, V. Colao, X. Qin, and S. M. Kang, "Strong convergence of the modified Mann iterative method for strict pseudo-contractions," Computers $\mathcal{E}$ Mathematics with Applications, vol. 57, no. 3, pp. 455-465, 2009.

[44] Y. Liu, "A general iterative method for equilibrium problems and strict pseudo-contractions in Hilbert spaces," Nonlinear Analysis: Theory, Methods E Applications, vol. 71, no. 10, pp. 4852-4861, 2009.

[45] R. T. Rockafellar, "Monotone operators and the proximal point algorithm," SIAM Journal on Control and Optimization, vol. 14, no. 5, pp. 877-898, 1976. 
[46] H.-K. Xu, "Viscosity approximation methods for nonexpansive mappings," Journal of Mathematical Analysis and Applications, vol. 298, no. 1, pp. 279-291, 2004.

[47] T. Suzuki, "Strong convergence of Krasnoselskii and Mann's type sequences for one-parameter nonexpansive semigroups without Bochner integrals," Journal of Mathematical Analysis and Applications, vol. 305, no. 1, pp. 227-239, 2005.

[48] J.-W. Peng and J.-C. Yao, “A new hybrid-extragradient method for generalized mixed equilibrium problems, fixed point problems and variational inequality problems," Taiwanese Journal of Mathematics, vol. 12, no. 6, pp. 1401-1432, 2008.

[49] B. Martinet, "Perturbation des méthodes d'optimisation. Applications," RAIRO Analyse Numérique, vol. 12, no. 2, pp. 153-171, 1978.

[50] M. C. Ferris, "Finite termination of the proximal point algorithm," Mathematical Programming, vol. 50, no. 3, pp. 359-366, 1991. 